\title{
Dimensions of the Boundaries of Self-Similar Sets
}

\author{
Ka-Sing Lau and Sze-Man Ngai
}

\section{CONTENTS}

1. Introduction

2. Boundary and Interior of $F$

3. The Finite Boundary Type Condition

4. Proof of Theorems $\mathbf{1 . 2}$ and $\mathbf{1 . 3}$

5. Examples on Computing Dimensions Acknowledgments

References

2000 AMS Subject Classification: Primary 28A78; Secondary 28A80

Keywords: Self-similar set, self-similar tile, self-affine tile, finite type condition, finite boundary type condition, Hausdorff dimension, box dimension.
We introduce a finite boundary type condition on iterated function systems of contractive similitudes on $\mathbb{R}^{d}$. Under this condition, we compute the Hausdorff dimension of the boundary of the attractor in terms of the spectral radius of some finite offspring matrix. We describe how to construct such a matrix. We also show that, in this case, the box dimension equals the Hausdorff dimension. In particular, this allows us to compute the Hausdorff dimension of the boundary of a class of self-similar sets defined by expansion matrices with noninteger entries.

\section{INTRODUCTION}

Let $\left\{\phi_{i}\right\}_{i=1}^{q}$ be an iterated function system (IFS) of contractive similitudes on $\mathbb{R}^{d}$ defined as

$$
\phi_{i}(x)=\rho_{i} R_{i} x+b_{i}, \quad i=1, \ldots, q,
$$

where $0<\rho_{i}<1$ is the contraction ratio, $R_{i}$ is an orthogonal transformation, and $b_{i} \in \mathbb{R}^{d}$. Let $F$ be the unique self-similar set (or attractor) defined by $\left\{\phi_{i}\right\}_{i=1}^{q}$. Then

$$
F=\bigcup_{i=1}^{q} \phi_{i}(F)
$$

(see, e.g., [Hutchinson 81], [Falconer 90]).

For a subset $E \subseteq \mathbb{R}^{d}$, let $E^{\circ}, \partial E$, and $\operatorname{diam}(E)$ denote, respectively, the interior, boundary, and diameter of $E$. Let $\operatorname{dim}_{H}(E), \operatorname{dim}_{B}(E)$, and $\mathcal{H}^{s}(E)$ be, respectively, the Hausdorff dimension, box dimension, and $s$-dimensional Hausdorff measure of $E$. We refer the reader to [Falconer 90] for these definitions. We also let $\# A$ denote the cardinality of a set $A$.

Much work has been done in computing the Hausdorff dimension of both the set $F$ and the boundary of $F$. The computation of the dimension of $\partial F$ is of particular interest in connection with self-affine and self-similar tiles. Suppose

$$
\phi_{i}(x)=A^{-1}\left(x+d_{i}\right), \quad d_{i} \in \mathcal{D},
$$

where $A$ is an expanding matrix (i.e., all eigenvalues are in modulus greater than 1) with integer entries and $\mathcal{D}$

(c) A K Peters, Ltd. $1058-6458 / 2001 \$ 0.50$ per page Experimental Mathematics 12:1, page 13 
is a finite set of vectors in $\mathbb{Z}^{d}$, called the digit set. Under the assumption that $A$ is conjugate to a similitude and $\left\{\phi_{i}\right\}_{i=1}^{q}$ satisfies the open set condition (see [Hutchinson 81], [Falconer 90]), algorithms have been obtained to compute the dimensions of $\partial F$ (see [Veerman 98], [Strichartz and Wang 99], [Kenyon et al. 99], and [Duvall et al. 00]). In this case, it is known that

$$
\operatorname{dim}_{H}(\partial F)=\operatorname{dim}_{B}(\partial F) \text { and } \mathcal{H}^{s}(\partial F)>0,
$$

where $s=\operatorname{dim}_{H}(\partial F)$ (see [Strichartz and Wang 99]). Recently, He, Lau, and Rao have extended the computations to allow $\# \mathcal{D}>|\operatorname{det}(A)|$ (see [He et al. 03]). A major difference in this case is that the open set condition fails. Their method also includes an algorithm to determine whether $F$ has a nonempty interior. [Dekking and van der Wal 02] have studied such an extension for a class of recurrent iterated function systems.

The main purpose of this paper is to extend the results on the dimensions of the boundary of a self-similar set to more general IFSs that do not necessarily satisfy (1-3), nor the open set condition. Our basic framework is the finite boundary type condition to be introduced in Section 3 . The $\phi_{i} \mathrm{~s}$ in our setting can have different contraction ratios and $\left(\rho_{i} R_{i}\right)^{-1}$ need not have integer entries. The finite boundary type condition is satisfied by a finite type IFS that possesses a finite type condition set containing the attractor. This allows us to include many interesting examples of IFSs with overlaps, in particular, the classes of examples in [Ngai and Wang 01, Theorems 2.7 and 2.9]. There are also IFSs that are of finite boundary type, but not of finite type. Our algorithm differs from the one in [He et al. 03] in that we do not make use of an auxiliary IFS, which is crucial there, but does not seem to exist for the IFSs in which we are mainly interested. An example of such an IFS is $S_{1}(x)=\rho x, S_{2}(x)=\rho x+(1-\rho)$, where $1 / 2<\rho<(\sqrt{5}-1) / 2$ (see Example 5.2).

We need some standard notation. Let $\Sigma_{q}^{n}=$ $\{1,2, \ldots, q\}^{n}$ and $\Sigma_{q}^{*}=\cup_{n \geq 0} \Sigma_{q}^{n}$ be the set of all finite words in $\Sigma_{q}$, where $\Sigma_{q}^{n}$ is the set of all words of length $n$ and $\Sigma_{q}^{0}$ contains only the empty word $\emptyset$. Let $\Sigma_{q}^{\mathbb{N}}$ denote the set of all infinite sequences $\left(i_{1}, i_{2}, \ldots\right)$ with $i_{k} \in \Sigma_{q}$. For $\mathbf{j} \in \Sigma_{q}^{n}$, let $|\mathbf{j}|=n$ denote the length of $\mathbf{j}$. For $\mathbf{i} \in \Sigma_{q}^{m}$ and $\mathbf{j} \in \Sigma_{q}^{n}$, let $\mathbf{i j} \in \Sigma_{q}^{m+n}$ be the concatenation of $\mathbf{i}$ with $\mathbf{j}$. For $\mathbf{j}=\left(j_{1}, \ldots, j_{n}\right) \in \Sigma_{q}^{n}$, we use the convenient notation

$\phi_{\mathbf{j}}:=\phi_{j_{1}} \circ \cdots \circ \phi_{j_{n}}, \quad \rho_{\mathbf{j}}:=\rho_{j_{1}} \cdots \rho_{j_{n}}, \quad R_{\mathbf{j}}:=R_{j_{1}} \circ \cdots \circ R_{j_{n}}$, with $\rho_{\emptyset}=1$ and $\phi_{\emptyset}=R_{\emptyset}:=$ identity. Let $\rho=\min \left\{\rho_{i}\right.$ : $1 \leq i \leq q\}$ and for all $k \geq 0$ define

$\Lambda_{k}:=\left\{\mathbf{j}=\left(j_{1}, \ldots, j_{n}\right) \in \Sigma_{q}^{*}: \rho_{\mathbf{j}} \leq \rho^{k}<\rho_{\left(j_{1}, \ldots, j_{n-1}\right)}\right\}$

$$
\begin{aligned}
\mathcal{V}_{k} & :=\left\{\left(\phi_{\mathbf{j}}, k\right): \mathbf{j} \in \Lambda_{k}\right\} \\
\mathcal{V} & :=\bigcup_{k=0}^{\infty} \mathcal{V}_{k} .
\end{aligned}
$$

We call each $\mathbf{v}=\left(\phi_{\mathbf{j}}, k\right) \in \mathcal{V}_{k}$ a vertex. Note that in [Ngai and Wang 01], a vertex $\left(\phi_{\mathbf{j}}, k\right)$ is denoted equivalently by $\left(\rho_{\mathbf{j}} R_{\mathbf{j}}, \phi_{\mathbf{j}}(0), k\right)$. For $\mathbf{v}=\left(\phi_{\mathbf{j}}, k\right) \in \mathcal{V}_{k}$, we use the convenient notation $\phi_{\mathbf{v}}:=\phi_{\mathbf{j}}$. Note also that $\Lambda_{0}$ contains only the empty word $\emptyset$ and $\mathcal{V}_{0}$ contains only the vertex $\mathbf{v}=\left(\phi_{\emptyset}, 0\right)$, with $\phi_{\emptyset}(E)=\phi_{\mathbf{v}}(E):=E$ for any $E \subseteq \mathbb{R}^{d}$. $\mathbf{v}=\left(\phi_{\emptyset}, 0\right)$ is call the root vertex and is denoted by $\mathbf{v}_{\text {root }}$.

Now let $\left\{\phi_{i}\right\}_{i=1}^{q}$ be defined as in (1-1). The following partition of $\mathcal{V}_{k}$ and $\mathcal{V}$ is similar to that in [He et al. 03]. But instead of utilizing an auxiliary system, we make use of a bounded open set $\Omega$ containing $F$ and invariant under $\left\{\phi_{i}\right\}_{i=1}^{q}$, i.e., $\bigcup_{i=1}^{q} \phi_{i}(\Omega) \subseteq \Omega$. Note that such an $\Omega$ always exists.

For $k \geq 0$, define

$$
\begin{aligned}
\mathcal{V}_{k}^{\circ}:= & \left\{\mathbf{v} \in \mathcal{V}_{k}: \exists \ell \in \mathbb{N}\right. \text { such that } \\
& \left.\phi_{\mathbf{v}}\left(\bigcup_{\mathbf{u} \in \mathcal{V}_{\ell}} \phi_{\mathbf{u}}(\Omega)\right) \subseteq \bigcup_{\mathbf{v}^{\prime} \in \mathcal{V}_{k^{\prime}}} \phi_{\mathbf{v}^{\prime}}(\Omega) \forall k^{\prime} \geq k\right\}, \\
\mathcal{V}_{k}^{\partial}:= & \mathcal{V}_{k} \backslash \mathcal{V}_{k}^{\circ}, \\
\mathcal{V}^{\circ}:= & \bigcup_{k=0}^{\infty} \mathcal{V}_{k}^{\circ}, \quad \mathcal{V}^{\partial}:=\bigcup_{k=0}^{\infty} \mathcal{V}_{k}^{\partial} .
\end{aligned}
$$

We remark that $\mathcal{V}_{k}^{\circ}, \mathcal{V}_{k}^{\partial}, \mathcal{V}^{\circ}$, and $\mathcal{V}^{\partial}$ depend on $\Omega$ and we will fix such an $\Omega$. Our first main result recovers a similar one in [He et al. 03].

Theorem 1.1. Suppose $\left\{\phi_{i}\right\}_{i=1}^{q}$ is an IFS of contractive similitudes on $\mathbb{R}^{d}$ as in (1-1). Let $\Omega$ be any invariant bounded open set containing $F$, and let $\mathcal{V}_{k}^{\circ}$ and $\mathcal{V}_{k}^{\partial}$ be defined with respect to $\Omega$ as in (1-5). Then

(a) $F^{\circ} \neq \emptyset$ if and only if $\mathcal{V}^{\circ} \neq \emptyset$;

(b) $F^{\circ}=\bigcup_{k=0}^{\infty} \bigcup_{\mathbf{v} \in \mathcal{V}_{k}^{\circ}} \phi_{\mathbf{v}}(F)$;

(c) $\partial F=\bigcap_{k=0}^{\infty} \bigcup_{\mathbf{v} \in \mathcal{V}_{k}^{\partial}} \phi_{\mathbf{v}}(\partial F)=\bigcap_{k=0}^{\infty} \bigcup_{\mathbf{v} \in \mathcal{V}_{k}^{\partial}} \phi_{\mathbf{v}}(F)=$ $\bigcap_{k=0}^{\infty} \bigcup_{\mathbf{v} \in \mathcal{V}_{k}^{\partial}} \phi_{\mathbf{v}}(\Omega)$

To compute the Hausdorff dimension of $\partial F$, we will introduce the notion of finite boundary type IFSs. Detailed 
definitions will be given in Section 3. Examples of finite boundary type IFSs will be provided in Sections 3 and 5 . Under the finite boundary type condition, we can compute $\operatorname{dim}_{H}(\partial F)$ by constructing a finite matrix $B$, called the boundary offspring matrix, to count $\# \mathcal{V}_{k}^{\partial}$. Details of the construction of $B$ will be described in Section 4 .

We remark here that in the construction of $B$, the definitions in (1-5) do not provide an effective algorithm for determining whether a given vertex belongs to $\mathcal{V}^{\circ}$ or $\mathcal{V}^{\partial}$. Propositions 2.2 and 2.3 provide a geometric criterion, but it is difficult to implement unless the geometry of $F$ is simple. Some other partial results to overcome this difficulty will be discussed in Section 5 . It would be very useful if an effective algebraic criterion can be found.

The main theorem below recovers all properties in (1-4). Moreover, in the case $F^{\circ}=\emptyset$, the algorithm computes $\operatorname{dim}_{H}(F)$, as in [He et al. 03].

Theorem 1.2. Assume that the IFS $\left\{\phi_{i}\right\}_{i=1}^{q}$ in (1-1) is of finite boundary type. Let $B$ be a corresponding boundary offspring matrix. Then

(a) $\operatorname{dim}_{H}(\partial F)=\operatorname{dim}_{B}(\partial F)=\frac{\ln \lambda_{B}}{-\ln \rho}$, where $\lambda_{B}$ is the spectral radius of $B$.

(b) $\mathcal{H}^{s}(\partial F)>0$ where $s=\operatorname{dim}_{H}(\partial F)$.

Theorem 1.2 is proved by first obtaining the box dimension of $\partial F$ in terms of the spectral radius of $B$. To obtain the result for the Hausdorff dimension, we use an argument in [Ngai and Wang 01] to define a mass distribution on $\partial F$ and prove Theorem 1.2(b), namely, $\mathcal{H}^{s}(\partial F)>0$ where $s=\operatorname{dim}_{B}(\partial F)$. From this, we deduce that $\operatorname{dim}_{H}(\partial F)=\operatorname{dim}_{B}(\partial F)$.

The problem of whether the Hausdorff dimension of $\partial F$ is strictly less than $d$ has been studied by Lagarias and Wang [1996] for self-affine tiles, by Keesling [1999] for self-similar sets, and by Lau and Xu [2000] for attractors of more general IFSs. For an IFS $\{\phi\}_{i=1}^{q}$ as defined in (1-1), it is proved in [Keesling 99, Theorem 2.1] and [Lau and $\mathrm{Xu} 00$, Corollary 1.3] that if $F^{\circ} \neq \emptyset$ and the similarity dimension of $\{\phi\}_{i=1}^{q}$ is $d$, i.e.,

$$
\sum_{i=1}^{q} \rho_{i}^{d}=1
$$

then $\operatorname{dim}_{H}(\partial F)<d$. In general, a finite type IFS does not satisfy (1-6). Nevertheless, the following result shows that the same conclusion holds.
Theorem 1.3. Let $\left\{\phi_{i}\right\}_{i=1}^{q}$ be a finite boundary type IFS of contractive similitudes on $\mathbb{R}^{d}$ with attractor $F$. Then $\operatorname{dim}_{H}(\partial F)<d$

Under the assumption of Theorem 1.3, $\operatorname{dim}_{H}(F)=d$ implies that $F^{\circ} \neq \emptyset$. In some of our calculations, this serves as a criterion to determine whether $F^{\circ}$ is empty.

In Section 2, we partition the vertices in $\mathcal{V}$ into $\mathcal{V}^{\circ}$ and $\mathcal{V}^{\partial}$ and prove Theorem 1.1. In Section 3, we introduce the finite boundary type condition and provide examples of IFSs satisfying this condition. In Section 4, we describe the construction of the boundary offspring matrix $B$. The rest of Section 4 is devoted to the proof of Theorems 1.2 and 1.3. In Section 5, we illustrate the algorithm by some examples.

\section{BOUNDARY AND INTERIOR OF $F$}

Throughout this section, we fix a bounded open set $\Omega$ containing the attractor $F$ and invariant under $\left\{\phi_{i}\right\}_{i=1}^{q}$, i.e., $\bigcup_{i=1}^{q} \phi_{i}(\Omega) \subseteq \Omega$. Let $\mathcal{V}_{k}^{\circ}, \mathcal{V}_{k}^{\partial}, \mathcal{V}^{\circ}$ and $\mathcal{V}^{\partial}$ be defined with respect to this fixed $\Omega$ as in $(1-5)$.

Proposition 2.1. Let $\left\{\phi_{i}\right\}_{i=1}^{q}$ be an IFS of contractive similitudes on $\mathbb{R}^{d}$ as defined in (1-1). Let $\Omega$ be a bounded open set containing $F$ and invariant under $\left\{\phi_{i}\right\}_{i=1}^{q}$. Then

(a) $\bigcup_{\mathbf{v} \in \mathcal{V}_{k}} \phi_{\mathbf{v}}(\Omega)$, where $k=0,1,2, \ldots$, is a decreasing sequence.

$$
\text { (b) } \begin{aligned}
F & =\bigcap_{k=0}^{\infty} \bigcup_{|\mathbf{i}|=k} \phi_{\mathbf{i}}(F)=\bigcap_{k=0}^{\infty} \bigcup_{\mathbf{v} \in \mathcal{V}_{k}} \phi_{\mathbf{v}}(F) \\
& =\bigcap_{k=0}^{\infty} \bigcup_{\mathbf{v} \in \mathcal{V}_{k}} \phi_{\mathbf{v}}(\Omega) .
\end{aligned}
$$

Proof: Part (a) follows easily from the invariance of $\Omega$ under $\left\{\phi_{i}\right\}_{i=1}^{q}$. The first two equalities in part (b) are well known. In fact, it follows by iterating (1-2) that

$$
F=\bigcup_{|\mathbf{i}|=k} \phi_{\mathbf{i}}(F)=\bigcup_{\mathbf{v} \in \mathcal{V}_{k}} \phi_{\mathbf{v}}(F) \quad \text { for all } k \geq 0 \text {. }
$$

To see the third equality, we let $x \in \bigcap_{k=0}^{\infty} \bigcup_{\mathbf{v} \in \mathcal{V}_{k}} \phi_{\mathbf{v}}(\Omega)$. Then for each $k \geq 0$, there exists some $\mathbf{v}_{k} \in \mathcal{V}_{k}$ such that $x \in \phi_{\mathbf{v}_{k}}(\Omega)$. But $\lim _{k \rightarrow \infty} d_{H}\left(\phi_{\mathbf{v}_{k}}(\bar{\Omega}), \phi_{\mathbf{v}_{k}}(F)\right)=$ 0 , where $d_{H}$ is the Hausdorff metric. Hence, $x \in F=$ $\bigcap_{k=0}^{\infty} \bigcup_{\mathbf{v} \in \mathcal{V}_{k}} \phi_{\mathbf{v}}(F)$. Thus

$$
\bigcap_{k=0}^{\infty} \bigcup_{\mathbf{v} \in \mathcal{V}_{k}} \phi_{\mathbf{v}}(F) \supseteq \bigcap_{k=0}^{\infty} \bigcup_{\mathbf{v} \in \mathcal{V}_{k}} \phi_{\mathbf{v}}(\Omega)
$$


The reverse inclusion is obvious and the proof is complete.

Proposition 2.2. Assume the same hypotheses of Proposition 2.1 and let $\mathbf{v} \in \mathcal{V}$. Then

$$
\mathbf{v} \in \mathcal{V}^{\circ} \Leftrightarrow \phi_{\mathbf{v}}(F) \subseteq F^{\circ} .
$$

Proof: Let $\mathbf{v} \in \mathcal{V}_{k}^{\circ}$. Using the definition of $\mathcal{V}_{k}^{\circ}$, there exists some $\ell$ such that

$\phi_{\mathbf{v}}(F) \subseteq \phi_{\mathbf{v}}\left(\bigcup_{\mathbf{u} \in \mathcal{V}_{\ell}} \phi_{\mathbf{u}}(\Omega)\right) \subseteq \bigcup_{\mathbf{v}^{\prime} \in \mathcal{V}_{k^{\prime}}} \phi_{\mathbf{v}^{\prime}}(\Omega) \quad$ for all $k^{\prime} \geq k$.

By Proposition 2.1(a), $\bigcup_{\mathbf{v}^{\prime} \in \mathcal{V}_{k^{\prime}}} \phi_{\mathbf{v}^{\prime}}(\Omega), k^{\prime}=0,1,2, \ldots$, is decreasing and hence, by using Proposition 2.1(b), we get

$$
\phi_{\mathbf{v}}\left(\bigcup_{\mathbf{u} \in \mathcal{V}_{\ell}} \phi_{\mathbf{u}}(\Omega)\right) \subseteq \bigcap_{k^{\prime}=0}^{\infty} \bigcup_{\mathbf{v}^{\prime} \in \mathcal{V}_{k^{\prime}}} \phi_{\mathbf{v}^{\prime}}(\Omega)=F .
$$

Now, since $\phi_{\mathbf{v}}\left(\cup_{\mathbf{u} \in \mathcal{V}_{\ell}} \phi_{\mathbf{u}}(\Omega)\right)$ is open, we have $\phi_{\mathbf{v}}\left(\cup_{\mathbf{u} \in \mathcal{V}_{\ell}} \phi_{\mathbf{u}}(\Omega)\right) \subseteq F^{\circ}$. Consequently, $\phi_{\mathbf{v}}(F) \subseteq F^{\circ}$.

For the converse, suppose $\phi_{\mathbf{v}}(F) \subseteq F^{\circ}$. Then by Proposition 2.1(b), we have

$$
\begin{aligned}
\phi_{\mathbf{v}}(F) & =\phi_{\mathbf{v}}\left(\bigcap_{k=0}^{\infty} \bigcup_{\mathbf{u} \in \mathcal{V}_{k}} \phi_{\mathbf{u}}(\Omega)\right) \\
& =\bigcap_{k=0}^{\infty} \phi_{\mathbf{v}}\left(\bigcup_{\mathbf{u} \in \mathcal{V}_{k}} \phi_{\mathbf{u}}(\Omega)\right) \subseteq F^{\circ} .
\end{aligned}
$$

Since $\phi_{\mathbf{v}}(F)$ is compact and $\phi_{\mathbf{v}}\left(\cup_{\mathbf{u} \in \mathcal{V}_{k}} \phi_{\mathbf{u}}(\Omega)\right)$, $k=0,1,2, \ldots$, is decreasing to $\phi_{\mathbf{v}}(F)$, there exists some $\ell$ such that

$$
\phi_{\mathbf{v}}\left(\bigcup_{\mathbf{u} \in \mathcal{V}_{\ell}} \phi_{\mathbf{u}}(\Omega)\right) \subseteq F^{\circ} \subseteq \bigcap_{k=0}^{\infty} \bigcup_{\mathbf{v} \in \mathcal{V}_{k}} \phi_{\mathbf{v}}(\Omega) .
$$

This implies that $\mathbf{v} \in \mathcal{V}^{\circ}$.

Proposition 2.3. Assume the same hypotheses of Proposition 2.1 and let $\mathbf{v} \in \mathcal{V}$. Then

$$
\mathbf{v} \in \mathcal{V}^{\partial} \Leftrightarrow \phi_{\mathbf{v}}(F) \cap \partial F \neq \emptyset .
$$

Proof: Using Proposition 2.2 and the fact that $\phi_{\mathbf{v}}(F) \subseteq$ $F$, we obtain the following equivalences:

$$
\begin{aligned}
\mathbf{v} \in \mathcal{V}^{\partial} & \Leftrightarrow \mathbf{v} \notin \mathcal{V}^{\circ} \\
& \Leftrightarrow \phi_{\mathbf{v}}(F) \nsubseteq F^{\circ} \quad \Leftrightarrow \quad \phi_{\mathbf{v}}(F) \cap \partial F \neq \emptyset .
\end{aligned}
$$

This proves the proposition.
By combining Propositions 2.1, 2.2, and 2.3, we can now prove Theorem 1.1.

Proof of Theorem 1.1: (a) If $F^{\circ} \neq \emptyset$, then it follows from Proposition 2.1(b) that there exists some $k \geq 0$ and $\mathbf{v} \in \mathcal{V}_{k}$ such that $\phi_{\mathbf{v}}(F) \subseteq F^{\circ}$. Proposition 2.2 now implies that $\mathbf{v} \in \mathcal{V}_{k}^{\circ}$ and thus, $\mathcal{V}^{\circ} \neq \emptyset$.

Conversely, assume $\mathcal{V}^{\circ} \neq \emptyset$ and let $\mathbf{v} \in \mathcal{V}_{k}^{\circ}$ for some $k \geq 0$. Then by Proposition 2.2 again, $\phi_{\mathbf{v}}(F) \subseteq F^{\circ}$ and therefore, $F^{\circ} \neq \emptyset$.

(b) It follows directly from Proposition 2.2 that

$$
\bigcup_{k=0}^{\infty} \bigcup_{\mathbf{v} \in \mathcal{V}_{k}^{\circ}} \phi_{\mathbf{v}}(F) \subseteq F^{\circ} .
$$

It remains to show the reverse inclusion. Let $x \in F^{\circ}$. Then by Proposition 2.1(b), for each $k$ sufficiently large, there exists some $\mathbf{v} \in \mathcal{V}_{k}$ such that $x \in \phi_{\mathbf{v}}(F) \subseteq F^{\circ}$. Proposition 2.2 now implies that $\mathbf{v} \in \mathcal{V}_{k}^{\circ}$ and therefore, $x \in \bigcup_{k=0}^{\infty} \bigcup_{\mathbf{v} \in \mathcal{V}_{k}^{\circ}} \phi_{\mathbf{v}}(F)$. This proves part (b).

(c) We first claim that

$$
\begin{aligned}
\partial F & \subseteq \bigcap_{k=0}^{\infty} \bigcup_{\mathbf{v} \in \mathcal{V}_{k}^{\partial}} \phi_{\mathbf{v}}(\partial F) \subseteq \bigcap_{k=0}^{\infty} \bigcup_{\mathbf{v} \in \mathcal{V}_{k}^{\partial}} \phi_{\mathbf{v}}(F) \\
& \subseteq \bigcap_{k=0}^{\infty} \bigcup_{\mathbf{v} \in \mathcal{V}_{k}^{\partial}} \phi_{\mathbf{v}}(\Omega) .
\end{aligned}
$$

It suffices to prove the first inclusion. To see this, we notice from (2-1) and Proposition 2.2 that for all $k \geq 0$,

$$
F=\left(\bigcup_{\mathbf{v} \in \mathcal{V}_{k}^{\circ}} \phi_{\mathbf{v}}(F)\right) \cup\left(\bigcup_{\mathbf{v} \in \mathcal{V}_{k}^{\partial}} \phi_{\mathbf{v}}(F)\right) \subseteq F^{\circ} \cup\left(\bigcup_{\mathbf{v} \in \mathcal{V}_{k}^{\partial}} \phi_{\mathbf{v}}(F)\right) .
$$

This implies that $\partial F \subseteq \bigcup_{\mathbf{v} \in \mathcal{V}_{k}^{\partial}} \phi_{\mathbf{v}}(F)$ for all $k \geq 0$. Hence, for $x \in \partial F$, there exists some $\mathbf{v} \in \mathcal{V}_{k}^{\partial}$ and $y \in F$ such that $x=\phi_{\mathbf{v}}(y)$. Obviously, $y$ must belong to $\partial F$. Thus, $x \in \cup_{\mathbf{v} \in \mathcal{V}_{k}^{\partial}} \phi_{\mathbf{v}}(\partial F)$ and the first inclusion in (2-2) follows. It remains to show

$$
\bigcap_{k=0}^{\infty} \bigcup_{\mathbf{v} \in \mathcal{V}_{k}^{\partial}} \phi_{\mathbf{v}}(\Omega) \subseteq \partial F .
$$

Let $x$ belong to the set on the left of the inclusion. Then for each $k \geq 0$, there exists some $\mathbf{v} \in \mathcal{V}_{k}^{\partial}$ such that $x \in$ $\phi_{\mathbf{v}}(\Omega)$. By Proposition 2.3, $\phi_{\mathbf{v}}(\Omega) \cap \partial F \neq \emptyset$. Let $y_{k} \in$ $\phi_{\mathbf{v}}(\Omega) \cap \partial F$. Then for each $k \geq 0$, we have

$$
\left|x-y_{k}\right| \leq \rho^{k} \operatorname{diam}(\Omega) .
$$

Consequently, $\left\{y_{k}\right\}$ is a sequence of points in $\partial F$ converging to $x$. Thus $x \in \partial F$. This proves (c) and completes the proof of Theorem 1.1. 


\section{THE FINITE BOUNDARY TYPE CONDITION}

In this section, we define the finite boundary type condition and provide some examples. We continue to use the notation introduced in Section 1.. For the reader's convenience, we will first recall the definition of the finite type condition (see [Ngai and Wang 01]).

Fix any nonempty bounded open set $\Omega$ which is invariant under $\left\{\phi_{i}\right\}_{i=1}^{q}(\Omega$ need not contain $F)$. Two vertices $\mathbf{v}, \mathbf{v}^{\prime} \in \mathcal{V}_{k}$ are neighbors (with respect to $\Omega$ ) if $\phi_{\mathbf{v}}(\Omega) \cap \phi_{\mathbf{v}^{\prime}}(\Omega) \neq \emptyset$. The set of vertices

$$
\Omega(\mathbf{v}):=\left\{\mathbf{v}^{\prime}: \mathbf{v}^{\prime} \text { is a neighbor of } \mathbf{v}\right\}
$$

is called the neighborhood of $\mathbf{v}$ (with respect to $\Omega$ ). Define an equivalence relation on $\mathcal{V}$. Two vertices $\mathbf{v} \in \mathcal{V}_{k}$ and $\mathbf{v}^{\prime} \in \mathcal{V}_{k^{\prime}}$ are equivalent, denoted by $\mathbf{v} \sim \mathbf{v}^{\prime}$ (or more precisely by $\mathbf{v} \sim_{\tau} \mathbf{v}^{\prime}$ ), if there exists a similitude $\tau$ : $\mathbb{R}^{d} \rightarrow \mathbb{R}^{d}$ (depending on $\mathbf{v}$ and $\mathbf{v}^{\prime}$ ) of the form $\tau(x)=$ $\rho^{k^{\prime}-k} U x+c$, where $U$ is orthogonal and $c \in \mathbb{R}^{d}$, such that

$\phi_{\mathbf{v}^{\prime}}=\tau \circ \phi_{\mathbf{v}}$ and $\left\{\phi_{\mathbf{u}^{\prime}}: \mathbf{u}^{\prime} \in \Omega\left(\mathbf{v}^{\prime}\right)\right\}=\left\{\tau \circ \phi_{\mathbf{u}}: \mathbf{u} \in \Omega(\mathbf{v})\right\}$.

We denote the equivalence class containing $\mathbf{v}$ by $[\mathbf{v}]:=$ $[\mathbf{v}]_{\Omega}$ and call it the neighborhood type of $\mathbf{v}$ (with respect to $\Omega$ ). (Note that in [Ngai and Wang 01], $\mathbf{v} \sim_{\tau} \mathbf{v}^{\prime}$ is denoted by $\Omega(\mathbf{v}) \sim_{\tau} \Omega\left(\mathbf{v}^{\prime}\right)$ and $[\mathbf{v}]_{\Omega}$ is denoted by $[\Omega(\mathbf{v})]$ instead.)

Recall that an IFS of the form (1-1) is said to be of finite type if there exists a nonempty bounded invariant open set $\Omega$ such that $\mathcal{N}:=\{[\mathbf{v}]: \mathbf{v} \in \mathcal{V}\}$ is a finite set. $\Omega$ is called a finite type condition set (or simply FT-set).

To describe the finite boundary type condition, we will fix a bounded open invariant set $\Omega$ for $\left\{\phi_{i}\right\}_{i=1}^{q}$ and assume in addition that $F \subseteq \Omega$. Note that such an $\Omega$ always exists. Let $\mathcal{V}_{k}^{\circ}, \mathcal{V}_{k}^{\partial}, \mathcal{V}^{\circ}, \mathcal{V}^{\partial}$ be defined with respect to this fixed $\Omega$ as in (1-5). It is a key property that if $\mathbf{v} \sim_{\tau} \mathbf{v}^{\prime}$, then $\mathbf{v} \in \mathcal{V}^{\partial}$ if and only if $\mathbf{v}^{\prime} \in \mathcal{V}^{\partial}$ (Proposition 3.3). This is proved by using Proposition 2.3 and by analyzing the image of $\phi_{\mathbf{v}}(F) \cap F$ under $\tau$. For $\mathbf{v} \in \mathcal{V}$, define

$\Omega^{e}(\mathbf{v}):=\left\{\mathbf{u} \in \Omega(\mathbf{v}) \backslash\{\mathbf{v}\}: \phi_{\mathbf{u}}(\partial F) \cap \phi_{\mathbf{v}}(\partial F) \cap F^{\circ} \neq \emptyset\right\}$.

Also, for $\mathbf{u} \in \Omega^{e}(\mathbf{v})$, define

$$
\partial F_{\mathbf{u}, \mathbf{v}}:=\left\{x \in \partial F: \phi_{\mathbf{u}}(x) \in \phi_{\mathbf{v}}(\partial F) \cap F^{\circ}\right\} .
$$

Then we have the following useful identity:

Proposition 3.1. Let $F$ be the attractor of an IFS $\left\{\phi_{i}\right\}_{i=1}^{q}$ of contractive similitudes on $\mathbb{R}^{d}$, and let $\Omega$ be a bounded open invariant set containing $F$. Then for any $\mathbf{v} \in \mathcal{V}$,

$$
\begin{aligned}
& \phi_{\mathbf{v}}(F) \cap \partial F= \\
& \phi_{\mathbf{v}}(\partial F) \backslash\left(\bigcup_{\mathbf{u} \in \Omega(\mathbf{v})} \phi_{\mathbf{u}}\left(F^{\circ}\right) \cup \bigcup_{\mathbf{u} \in \Omega^{e}(\mathbf{v})} \phi_{\mathbf{u}}\left(\partial F_{\mathbf{u}, \mathbf{v}}\right)\right) .
\end{aligned}
$$

Proof: Let $x \in \phi_{\mathbf{v}}(F) \cap \partial F$. Then $x \in \phi_{\mathbf{v}}(\partial F)$ because $\phi_{\mathbf{v}}\left(F^{\circ}\right) \subseteq F^{\circ}$. Since $\bigcup_{\mathbf{u} \in \Omega(\mathbf{v})} \phi_{\mathbf{u}}\left(F^{\circ}\right) \cup$ $\bigcup_{\mathbf{u} \in \Omega^{e}(\mathbf{v})} \phi_{\mathbf{u}}\left(\partial F_{\mathbf{u}, \mathbf{v}}\right) \subseteq F^{\circ}, x$ must belong to the set on the right side of $(3-1)$.

Conversely, let $x$ belong to the set on the right side of (3-1). Then $x \in \phi_{\mathbf{v}}(\partial F) \subseteq \phi_{\mathbf{v}}(F)$. To show that $x \in \partial F$, let $x=\phi_{\mathbf{v}}(y)$ where $y \in \partial F$. Consider the following two cases.

Case 1. $x \in \bigcup_{\mathbf{u} \in \Omega(\mathbf{v}) \backslash\{\mathbf{v}\}} \phi_{\mathbf{u}}(F)$. In this case, $x \in \cup_{\mathbf{u} \in \Omega(\mathbf{v}) \backslash\{\mathbf{v}\}} \phi_{\mathbf{u}}(\partial F)$ since $x \notin \cup_{\mathbf{u} \in \Omega(\mathbf{v}) \backslash\{\mathbf{v}\}} \phi_{\mathbf{u}}\left(F^{\circ}\right)$. Hence, $x=\phi_{\mathbf{u}}(w)$ for some $\mathbf{u} \in \Omega(\mathbf{v}) \backslash\{\mathbf{v}\}$ and some $w \in \partial F$. But $x \notin \bigcup_{\mathbf{u} \in \Omega^{e}(\mathbf{v})} \phi_{\mathbf{u}}\left(\partial F_{\mathbf{u}, \mathbf{v}}\right)$ implies that $\{x\} \cap F^{\circ}=\emptyset$. Consequently, $x \in \partial F$.

Case 2. $x \notin \bigcup_{\mathbf{u} \in \Omega(\mathbf{v}) \backslash\{\mathbf{v}\}} \phi_{\mathbf{u}}(F)$. In this case, $x \in \partial F$ because $y \in \partial F, \phi_{\mathbf{v}}$ is a similitude, and there are no overlapping $\phi_{\mathbf{u}}(F)$ in a sufficiently small neighborhood of $x$.

Proposition 3.2. Let $\left\{\phi_{i}\right\}_{i=1}^{q}$ be an IFS of contractive similitudes on $\mathbb{R}^{d}$ with attractor $F$, and let $\Omega$ be a bounded open invariant set containing $F$. Assume $\mathbf{v} \in \mathcal{V}_{k}, \mathbf{v}^{\prime} \in \mathcal{V}_{k^{\prime}}$, and $\mathbf{v} \sim_{\tau} \mathbf{v}^{\prime}$. Let $\mathbf{u} \in \Omega(\mathbf{v})$ and $\mathbf{u}^{\prime} \in \Omega\left(\mathbf{v}^{\prime}\right)$ satisfy $\phi_{\mathbf{u}^{\prime}}=\tau \circ \phi_{\mathbf{u}}$. Then

(a) $\mathbf{u} \in \Omega^{e}(\mathbf{v})$ if and only if $\mathbf{u}^{\prime} \in \Omega^{e}\left(\mathbf{v}^{\prime}\right)$.

(b) $\partial F_{\mathbf{u}, \mathbf{v}}=\partial F_{\mathbf{u}^{\prime}, \mathbf{v}^{\prime}}$.

Proof: (a) Let $\mathbf{u} \in \Omega^{e}(\mathbf{v})$. Then $\phi_{\mathbf{u}}(\partial F) \cap \phi_{\mathbf{v}}(\partial F) \cap F^{\circ} \neq$ $\emptyset$. Hence, there exist some $z \in \phi_{\mathbf{u}}(\partial F) \cap \phi_{\mathbf{v}}(\partial F)$ and a ball $B_{\delta}(z)$ such that

$$
B_{\delta}(z) \subseteq \bigcup_{\mathbf{w} \in \Omega(\mathbf{v})} \phi_{\mathbf{w}}(F) \subseteq F .
$$

Write $z=\phi_{\mathbf{u}}(x)=\phi_{\mathbf{v}}(y)$ with $x, y \in \partial F$. Then

$$
\begin{aligned}
& \phi_{\mathbf{u}}(x) \in \phi_{\mathbf{u}}(\partial F) \cap \phi_{\mathbf{v}}(\partial F) \\
& \quad \Rightarrow \tau \circ \phi_{\mathbf{u}}(x) \in \tau \circ \phi_{\mathbf{u}}(\partial F) \cap \tau \circ \phi_{\mathbf{v}}(\partial F) .
\end{aligned}
$$

Moreover, by (3-2), we have

$$
B_{\delta}\left(\phi_{\mathbf{u}}(x)\right)=B_{\delta}\left(\phi_{\mathbf{v}}(y)\right) \subseteq \bigcup_{\mathbf{w} \in \Omega(\mathbf{v})} \phi_{\mathbf{w}}(F) \subseteq F .
$$


Let $r$ be the similarity ratio of $\tau$. Then we have $\phi_{\mathbf{u}^{\prime}}(x) \in$ $\phi_{\mathbf{u}^{\prime}}(\partial F) \cap \phi_{\mathbf{v}^{\prime}}(\partial F)$ and

$$
B_{r \delta}\left(\phi_{\mathbf{u}^{\prime}}(x)\right)=B_{r \delta}\left(\phi_{\mathbf{v}^{\prime}}(y)\right) \subseteq \bigcup_{\mathbf{w}^{\prime} \in \Omega\left(\mathbf{v}^{\prime}\right)} \phi_{\mathbf{w}^{\prime}}(F) \subseteq F .
$$

(The first inclusion above is because $\mathbf{w} \in \Omega(\mathbf{v})$ if and only if $\mathbf{w}^{\prime} \in \Omega\left(\mathbf{v}^{\prime}\right)$ where $\phi_{\mathbf{w}^{\prime}}=\tau \circ \phi_{\mathbf{w}}$.) This implies that $\phi_{\mathbf{u}^{\prime}}(x) \in \phi_{\mathbf{u}^{\prime}}(\partial F) \cap \phi_{\mathbf{v}^{\prime}}(\partial F) \cap F^{\circ}$ and therefore $\phi_{\mathbf{u}^{\prime}}(\partial F) \cap$ $\phi_{\mathbf{v}^{\prime}}(\partial F) \cap F^{\circ} \neq \emptyset$. Hence, $\mathbf{u}^{\prime} \in \Omega^{e}\left(\mathbf{v}^{\prime}\right)$. The converse follows by symmetry.

(b) Again let $r$ denote the similarity ratio of $\tau$. Let $x \in \partial F_{\mathbf{u}, \mathbf{v}}$. Then

$$
\begin{aligned}
x \in & x F \text { and } \phi_{\mathbf{u}}(x) \in \phi_{\mathbf{v}}(\partial F) \cap F^{\circ} \\
\Rightarrow \quad & x \in \partial F, \phi_{\mathbf{u}}(x) \in \phi_{\mathbf{v}}(\partial F) \quad \text { and there is a ball } \\
& B_{\delta}\left(\phi_{\mathbf{u}}(x)\right) \subseteq \bigcup_{\mathbf{w} \in \Omega(\mathbf{v})} \phi_{\mathbf{w}}(F) \subseteq F \\
\Rightarrow \quad & x \in \partial F, \tau \circ \phi_{\mathbf{u}}(x) \in \tau \circ \phi_{\mathbf{v}}(\partial F) \text { and } \\
& \tau\left(B_{\delta}\left(\phi_{\mathbf{u}}(x)\right)\right) \subseteq \bigcup_{\mathbf{w} \in \Omega(\mathbf{v})} \tau \circ \phi_{\mathbf{w}}(F) \\
\Rightarrow \quad & x \in \partial F, \phi_{\mathbf{u}^{\prime}}(x) \in \phi_{\mathbf{v}^{\prime}}(\partial F) \text { and } \\
& B_{r \delta}\left(\phi_{\mathbf{u}^{\prime}}(x)\right) \subseteq \bigcup_{\mathbf{w}^{\prime} \in \Omega\left(\mathbf{v}^{\prime}\right)} \phi_{\mathbf{w}^{\prime}}(F) \subseteq F \\
\Rightarrow \quad & x \in \partial F \text { and } \phi_{\mathbf{u}^{\prime}}(x) \in \phi_{\mathbf{v}^{\prime}}(\partial F) \cap F^{\circ} \\
\Rightarrow \quad & x \in \partial F_{\mathbf{u}^{\prime}, \mathbf{v}^{\prime}} .
\end{aligned}
$$

Thus, $\partial F_{\mathbf{u}, \mathbf{v}} \subseteq \partial F_{\mathbf{u}^{\prime}, \mathbf{v}^{\prime}}$. The reverse inclusion follows by symmetry and this completes the proof of the proposition.

Proposition 3.3. Assume the same hypotheses of Proposition 3.2. Then

$$
\tau\left(\phi_{\mathbf{v}}(F) \cap \partial F\right)=\phi_{\mathbf{v}^{\prime}}(F) \cap \partial F .
$$

Consequently, $\mathbf{v} \in \mathcal{V}^{\partial}$ if and only if $\mathbf{v}^{\prime} \in \mathcal{V}^{\partial}$.

Proof: Equality (3-1) implies that

$$
\begin{aligned}
& \tau\left(\phi_{\mathbf{v}}(F) \cap \partial F\right)= \\
& \tau \circ \phi_{\mathbf{v}}(\partial F) \backslash\left(\bigcup_{\mathbf{u} \in \Omega(\mathbf{v})} \tau \circ \phi_{\mathbf{u}}\left(F^{\circ}\right) \cup \bigcup_{\mathbf{u} \in \Omega^{e}(\mathbf{v})} \tau \circ \phi_{\mathbf{u}}\left(\partial F_{\mathbf{u}, \mathbf{v}}\right)\right) .
\end{aligned}
$$

Since $\mathbf{u} \in \Omega(\mathbf{v})$ if and only if $\mathbf{u}^{\prime} \in \Omega\left(\mathbf{v}^{\prime}\right)$ where $\phi_{\mathbf{u}^{\prime}}=$ $\tau \circ \phi_{\mathbf{u}}$, we have

$$
\bigcup_{\mathbf{u} \in \Omega(\mathbf{v})} \tau \circ \phi_{\mathbf{u}}\left(F^{\circ}\right)=\bigcup_{\mathbf{u}^{\prime} \in \Omega\left(\mathbf{v}^{\prime}\right)} \phi_{\mathbf{u}^{\prime}}\left(F^{\circ}\right) .
$$

Next, by using Proposition 3.2, we have

$$
\bigcup_{\mathbf{u} \in \Omega^{e}(\mathbf{v})} \tau \circ \phi_{\mathbf{u}}\left(\partial F_{\mathbf{u}, \mathbf{v}}\right)=\bigcup_{\mathbf{u}^{\prime} \in \Omega^{e}\left(\mathbf{v}^{\prime}\right)} \phi_{\mathbf{u}^{\prime}}\left(\partial F_{\mathbf{u}^{\prime}, \mathbf{v}^{\prime}}\right) .
$$

Lastly, combining Equations (3-3), (3-4), (3-5), and Proposition 3.1 yields

$$
\begin{aligned}
& \tau\left(\phi_{\mathbf{v}}(F) \cap \partial F\right)= \\
& \phi_{\mathbf{v}^{\prime}}(\partial F) \backslash\left(\bigcup_{\mathbf{u}^{\prime} \in \Omega\left(\mathbf{v}^{\prime}\right)} \phi_{\mathbf{u}^{\prime}}\left(F^{\circ}\right) \cup \bigcup_{\mathbf{u}^{\prime} \in \Omega^{e}\left(\mathbf{v}^{\prime}\right)} \phi_{\mathbf{u}^{\prime}}\left(\partial F_{\mathbf{u}^{\prime}, \mathbf{v}^{\prime}}\right)\right) \\
& =\phi_{\mathbf{v}^{\prime}}(F) \cap \partial F .
\end{aligned}
$$

This proves the stated equality. The last assertion of the proposition follows by combining this result with Proposition 2.3.

We call

$$
\mathcal{N}^{\circ}:=\left\{[\mathbf{v}]: \mathbf{v} \in \mathcal{V}^{\circ}\right\} \quad \text { and } \quad \mathcal{N}^{\partial}:=\left\{[\mathbf{v}]: \mathbf{v} \in \mathcal{V}^{\partial}\right\}
$$

the collections of all interior neighborhood types and boundary neighborhood types, respectively. According to Proposition 3.3, $\mathcal{N}=\mathcal{N}^{\circ} \cup \mathcal{N}^{\partial}$ and $\mathcal{N}^{\circ} \cap \mathcal{N}^{\partial}=\emptyset$.

Let $\mathcal{G}$ and $\mathcal{G}_{R}$ be the infinite graphs defined in [Ngai and Wang 01] as follows. Both $\mathcal{G}$ and $\mathcal{G}_{R}$ have $\mathcal{V}$ as the set of all vertices. The edges in $\mathcal{G}$ are defined as follows. Let $\mathbf{v} \in \mathcal{V}_{k}$ and $\mathbf{v}^{\prime} \in \mathcal{V}_{k+1}$. Suppose there exist $\mathbf{j} \in \Lambda_{k}, \mathbf{j}^{\prime} \in \Lambda_{k+1}$, and $\mathbf{k} \in \Sigma_{q}^{*}$ such that

$$
\mathbf{v}=\left(\phi_{\mathbf{j}}, k\right), \quad \mathbf{v}^{\prime}=\left(\phi_{\mathbf{j}^{\prime}}, k+1\right), \quad \text { and } \quad \mathbf{j}^{\prime}=\mathbf{j k} .
$$

Then we connect a directed edge $\mathbf{k}: \mathbf{v} \rightarrow \mathbf{v}^{\prime}$. We call $\mathbf{v}$ a parent of $\mathbf{v}^{\prime}$ and $\mathbf{v}^{\prime}$ an offspring (or descendant) of $\mathbf{v}$.

The reduced graph $\mathcal{G}_{R}$ is obtained from $\mathcal{G}$ by removing all but the smallest (in the lexicographical order) directed edge going to a vertex.

We introduce the graphs $\mathcal{G}^{\partial}$ and $\mathcal{G}_{R}^{\partial}$. The vertex set for both graphs is $\mathcal{V}^{\partial}$ and the directed edges in $\mathcal{G}^{\partial}$ and $\mathcal{G}_{R}^{\partial}$ are the restrictions of the edges in $\mathcal{G}$ and $\mathcal{G}_{R}$, respectively, to $\mathcal{V}^{\partial}$.

The following proposition says that equivalent boundary vertices generate the same number of offspring of each boundary neighborhood type.

Proposition 3.4. Let $\mathbf{v} \in \mathcal{V}_{k}^{\partial}$ and $\mathbf{v}^{\prime} \in \mathcal{V}_{k^{\prime}}^{\partial}$ such that $[\mathbf{v}]=\left[\mathbf{v}^{\prime}\right]$. Let $\mathbf{u}_{1}, \ldots, \mathbf{u}_{m} \in \mathcal{V}_{k+1}^{\partial}$ be the offspring of $\mathbf{v}$ and let $\mathbf{u}_{1}^{\prime}, \ldots, \mathbf{u}_{\ell}^{\prime} \in \mathcal{V}_{k^{\prime}+1}^{\partial}$ be the offspring of $\mathbf{v}^{\prime}$ in $\mathcal{G}_{R}^{\partial}$. Then

$$
\left\{\left[\mathbf{u}_{i}\right]: 1 \leq i \leq m\right\}=\left\{\left[\mathbf{u}_{i}^{\prime}\right]: 1 \leq i \leq \ell\right\}
$$

counting multiplicity. In particular, $m=\ell$. 
Proof: Let $\mathbf{u}_{1}, \ldots, \mathbf{u}_{m}, \mathbf{u}_{m+1}, \ldots, \mathbf{u}_{s}$ be all the offspring of $\mathbf{v}$ in $\mathcal{G}_{R}$, with $\mathbf{u}_{1}, \ldots, \mathbf{u}_{m} \in \mathcal{V}_{k+1}^{\partial}$ and $\mathbf{u}_{m+1}, \ldots, \mathbf{u}_{s} \in$ $\mathcal{V}_{k+1}^{\circ}$. Similarly, let $\mathbf{u}_{1}^{\prime}, \ldots, \mathbf{u}_{\ell}^{\prime}, \mathbf{u}_{\ell+1}^{\prime}, \ldots, \mathbf{u}_{s}^{\prime}$ be all the offspring of $\mathbf{v}^{\prime}$ in $\mathcal{G}_{R}$, with $\mathbf{u}_{1}^{\prime}, \ldots, \mathbf{u}_{\ell}^{\prime} \in \mathcal{V}_{k^{\prime}+1}^{\partial}$ and $\mathbf{u}_{\ell+1}^{\prime}, \ldots, \mathbf{u}_{s}^{\prime} \in \mathcal{V}_{k^{\prime}+1}^{\circ}$. Let $\mathbf{v} \sim_{\tau} \mathbf{v}^{\prime}$ with $\tau \circ \phi_{\mathbf{u}_{i}}=\phi_{\mathbf{u}_{i}^{\prime}}$ for all $1 \leq i \leq s$. Then by [Ngai and Wang 01, Proposition $2.2]$

$$
\left[\mathbf{u}_{i}\right]=\left[\mathbf{u}_{i}^{\prime}\right] \quad \text { for all } \quad 1 \leq i \leq s .
$$

By Proposition 3.3,

$$
\mathbf{u}_{i} \in \mathcal{V}_{k+1}^{\partial} \quad \text { if and only if } \quad \mathbf{u}_{i}^{\prime} \in \mathcal{V}_{k^{\prime}+1}^{\partial}
$$

Consequently, $m=\ell$. This proves the proposition.

Definition 3.5. An IFS $\left\{\phi_{i}\right\}_{i=1}^{q}$ of contractive similitudes on $\mathbb{R}^{d}$ with attractor $F$ is said to be of finite boundary type if there exists a bounded invariant open set $\Omega$ containing $F$ such that $\mathcal{N}^{\partial}$ is a finite set. In this case, we say that $\Omega$ is a finite boundary type condition set (or simply an FBT-set).

The following proposition follows easily from definitions.

Proposition 3.6. Let $\left\{\phi_{i}\right\}_{i=1}^{q}$ be a finite type IFS of contractive similitudes on $\mathbb{R}^{d}$. Assume that there exists an FT-set $\Omega$ containing $F$. Then $\left\{\phi_{i}\right\}_{i=1}^{q}$ is of finite boundary type.

Remark 3.7. A finite boundary type IFS is not necessarily of finite type. We will illustrate this in Example 5.2. We do not know whether each finite type IFS is of finite boundary type.

Combining Proposition 3.6 and [Ngai and Wang 01, Theorems 2.7 and 2.9], we obtain the following classes of finite boundary type IFSs.

Let $M_{d}(\mathbb{R})$ and $M_{d}(\mathbb{Z})$ be the sets of all $d \times d$ matrices with entries in $\mathbb{R}$ and $\mathbb{Z}$, respectively.

Example 3.8. Let $\left\{\phi_{i}\right\}_{i=1}^{q}$ be an IFS of contractive similitudes on $\mathbb{R}^{d}$ of the form

$$
\phi_{i}(x)=A^{n_{i}} x+b_{i}, \quad 1 \leq i \leq q,
$$

where $A$ is a contractive similitude in $M_{d}(\mathbb{R})$ and $n_{i} \in$ $\mathbb{N}$. Assume that $A^{-1} \in M_{d}(\mathbb{Z})$ and all $b_{i} \in \mathbb{Z}^{d}$. Then $\left\{\phi_{i}\right\}_{i=1}^{q}$ is of finite boundary type and any bounded open invariant set containing $F$ is an FBT-set.

Example 3.8 generalizes the IFSs studied in [Veerman 98], [Kenyon et al. 99], [Strichartz and Wang 99], and
[Duvall et al. 00], as well as the self-similar ones in [He et al. 03]. The following example allows us to include another interesting family of finite boundary type IFSs. Recall that a Pisot number is an algebraic integer greater than 1 whose algebraic conjugates are all in modulus less than one. Let $\mathbb{Z}[x]$ denote the ring of polynomials over the integers $\mathbb{Z}$.

Example 3.9. Let $\left\{\phi_{i}\right\}_{i=1}^{q}$ be an IFS of contractive similitudes on $\mathbb{R}^{d}$ of the form

$$
\phi_{i}(x)=r^{n_{i}} R_{i} x+b_{i}, \quad 1 \leq i \leq q,
$$

where $r^{-1}$ is a Pisot number and for $1 \leq i \leq q, n_{i} \in \mathbb{N}$ and $R_{i}$ is orthogonal. Assume that $\left\{R_{i}\right\}_{i=1}^{q}$ generates a finite group $G$, and

$$
G\left\{b_{i}: 1 \leq i \leq q\right\} \subseteq c_{1} \mathbb{Z}\left[r^{-1}\right] \times \cdots \times c_{d} \mathbb{Z}\left[r^{-1}\right]
$$

for some $c_{1}, \ldots, c_{d} \in \mathbb{R}$. Then $\left\{\phi_{i}\right\}_{i=1}^{q}$ is of finite boundary type and any bounded open invariant set containing $F$ is an FBT-set.

\section{PROOF OF THEOREMS $\mathbf{1 . 2}$ and $\mathbf{1 . 3}$}

We begin by stating two lemmas without proof. The following lemma can be obtained as [Ngai and Wang 01, Lemma 3.1].

Lemma 4.1. Suppose $\left\{\phi_{i}\right\}_{i=1}^{q}$ is a finite boundary type IFS of contractive similitudes on $\mathbb{R}^{d}$ and let $\mathcal{V}^{\partial}$ be defined with respect to an FBT-set $\Omega$. Then for any positive constants $K_{1}, K_{2}$, there exists a positive integer $M=$ $M\left(K_{1}, K_{2}\right)$ such that for any integer $k \geq 0$ and any subsets $U, E \subseteq \mathbb{R}^{d}$ with $\operatorname{diam}(U) \leq K_{1} \rho^{k}$ and $E \subseteq B_{K_{2}}(0)$, we have

$$
\#\left\{\mathbf{v} \in \mathcal{V}_{k}^{\partial}: \phi_{\mathbf{v}}(E) \cap U \neq \emptyset\right\} \leq M
$$

Using Lemma 4.1 and a similar argument as in [Ngai and Wang 01, Lemma 3.2], we have

Lemma 4.2. Assume that $\left\{\phi_{i}\right\}_{i=1}^{q}$ as given in (1-1) is a finite boundary type IFS of contractive similitudes on $\mathbb{R}^{d}$ with attractor $F$. Let $\mathcal{V}_{k}^{\partial}$ be defined with respect to an FBT-set $\Omega$. Then

$$
\begin{aligned}
\liminf _{k \rightarrow \infty} \frac{\ln \# \mathcal{V}_{k}^{\partial}}{-k \ln \rho} & \leq \underline{\operatorname{dim}}_{B}(\partial F) \\
& \leq \overline{\operatorname{dim}}_{B}(\partial F) \leq \limsup _{k \rightarrow \infty} \frac{\ln \# \mathcal{V}_{k}^{\partial}}{-k \ln \rho}
\end{aligned}
$$


In view of Lemma 4.2, we need to evaluate $\# \mathcal{V}_{k}^{\partial}$. We achieve this by using a matrix $B$. The matrix $B$ has rows and columns indexed by the boundary neighborhood types. Suppose that the IFS (1-1) is of finite boundary type and let $\Omega$ be an FBT-set. We label the boundary neighborhood types as $\left\{\mathcal{T}_{1}^{\partial}, \ldots, \mathcal{T}_{N}^{\partial}\right\}$. The entries of $B=\left\{b_{i j}\right\}$ are defined as follows. For any $1 \leq i \leq N$, take a vertex $\mathbf{v} \in \mathcal{V}^{\partial}$ of $\mathcal{G}_{R}^{\partial}$ such that $[\mathbf{v}]=\overline{\mathcal{T}}_{i}^{\partial}$. Let $\mathbf{u}_{1}, \ldots, \mathbf{u}_{n}$ be the offspring of $\mathbf{v}$ in $\mathcal{G}_{R}^{\partial}$. Then

$$
b_{i j}=\#\left\{\ell: 1 \leq \ell \leq n,\left[\mathbf{u}_{\ell}\right]=\mathcal{T}_{j}^{\partial}\right\} .
$$

By Proposition 3.4, $b_{i j}$ is independent of the choice of the vertex $\mathbf{v}$.

Definition 4.3. We call the matrix $B$ the boundary offspring matrix of the finite boundary type IFS (1-1) with respect to $\Omega$.

We label the boundary neighborhood type of $\left[\mathbf{v}_{\text {root }}\right]$ as $\mathcal{T}_{1}^{\partial}$. Then

$$
\# \mathcal{V}_{k}^{\partial}=\mathbf{e}_{1}^{T} B^{k} \mathbf{1},
$$

where $\mathbf{1}=[1, \ldots, 1]^{T}$ and $\mathbf{e}_{1}=[1,0, \ldots, 0]^{T}$ are vectors in $\mathbb{R}^{N}$.

Theorem 4.4. Let $\left\{\phi_{i}\right\}_{i=1}^{q}$ be a finite boundary type IFS of contractive similitudes on $\mathbb{R}^{d}$ with attractor $F$. Then

$$
\underline{\operatorname{dim}}_{B}(\partial F)=\overline{\operatorname{dim}}_{B}(\partial F)=\operatorname{dim}_{B}(\partial F)=\frac{\ln \lambda_{B}}{-\ln \rho},
$$

where $\lambda_{B}$ is the spectral radius of the boundary offspring matrix $B$ (with respect to $\Omega$ ).

Proof: The proof is standard; we only include a brief sketch. Since all vertices in $\mathcal{G}_{R}^{\partial}$ are descendants of $\mathbf{v}_{\text {root }}$, we have

$$
\lim _{k \rightarrow \infty}\left(\mathbf{e}_{1}^{T} B^{k} \mathbf{1}\right)^{1 / k}=\lambda_{B}
$$

Hence, for all $\delta>0$ sufficiently small and all integers $k$ sufficiently large, we have

$$
\left(\lambda_{B}-\delta\right)^{k}<\mathbf{e}_{1}^{T} B^{k} \mathbf{1}<\left(\lambda_{B}+\delta\right)^{k} .
$$

Combining this with (4-1) and Lemma 4.2, we get

$$
\frac{\ln \left(\lambda_{B}-\delta\right)}{-\ln \rho} \leq \underline{\operatorname{dim}}_{B}(\partial F) \leq \overline{\operatorname{dim}}_{B}(\partial F) \leq \frac{\ln \left(\lambda_{B}+\delta\right)}{-\ln \rho} .
$$

The result follows by letting $\delta \rightarrow 0$.

Theorem 4.4 shows that $\ln \lambda_{B} /(-\ln \rho)$ is an upper bound for $\operatorname{dim}_{H}(\partial F)$. We will show that it is also a lower bound. Define a path in $\mathcal{G}_{R}^{\partial}$ to be an infinite sequence $\left(\mathbf{v}_{0}=\mathbf{v}_{\text {root }}, \mathbf{v}_{1}, \mathbf{v}_{2}, \ldots\right)$ such that $\mathbf{v}_{j} \in \mathcal{V}_{j}^{\partial}$ for all $j \geq 0$ and $\mathbf{v}_{j+1}$ is an offspring of $\mathbf{v}_{j}$ in $\mathcal{G}_{R}^{\partial}$. Let $\mathcal{P}^{\partial}$ denote the set of all paths in $\mathcal{G}_{R}^{\partial}$. For given vertices $\mathbf{v}_{0}=\mathbf{v}_{\text {root }}, \mathbf{v}_{1}, \ldots, \mathbf{v}_{k}$ such that $\mathbf{v}_{j+1}$ is an offspring of $\mathbf{v}_{j}$ in $\mathcal{G}_{R}^{\partial}$, we call the set

$\mathcal{I}_{\mathbf{v}_{0}, \mathbf{v}_{1}, \ldots, \mathbf{v}_{k}}:=\left\{\left(\mathbf{u}_{0}, \mathbf{u}_{1}, \ldots\right) \in \mathcal{P}^{\partial}: \mathbf{u}_{j}=\mathbf{v}_{j}\right.$ for $\left.0 \leq j \leq k\right\}$

a branch. It follows from the definition of $\mathcal{G}_{R}^{\partial}$ that the path from $\mathbf{v}_{0}$ to $\mathbf{v}_{k}$ is unique. We can therefore denote

$$
\mathcal{I}_{\mathbf{v}_{k}}:=\mathcal{I}_{\mathbf{v}_{0}, \mathbf{v}_{1}, \ldots, \mathbf{v}_{k}} .
$$

Theorem 4.5. Let $\left\{\phi_{i}\right\}_{i=1}^{q}$ be a finite boundary type IFS of contractive similitudes on $\mathbb{R}^{d}$ and let $s=\operatorname{dim}_{B}(\partial F)$. Then $\mathcal{H}^{s}(\partial F)>0$.

Proof: The proof is similar to that of [Ngai and Wang 01, Theorem 1.2]; we outline it here for completeness.

We first construct a mass distribution $\mu$ on $\mathcal{P}^{\partial}$ using the branches. Let $\mathcal{T}_{1}^{\partial}, \ldots, \mathcal{T}_{N}^{\partial}$ be the set of all the distinct boundary neighborhood types with $\mathcal{T}_{1}^{\partial}$ being the neighborhood type of $\left[\mathbf{v}_{\text {root }}\right]$. Let $B$ be the corresponding boundary offspring matrix with respect to $\Omega$. By Theorem 4.4, we have

$$
s=\frac{\ln \lambda_{B}}{-\ln \rho},
$$

where $\lambda_{B}$ is the spectral radius of $B$. Since all vertices in $\mathcal{G}_{R}^{\partial}$ are descendants of $\mathbf{v}_{\text {root }}$ and all boundary neighborhood types are generated by $\mathcal{T}_{1}^{\partial}$, we may find a $\lambda_{B^{-}}$ eigenvector $\mathbf{x}=\left[c_{1}, \ldots, c_{N}\right]^{T}$ of $B$ such that $c_{1}>0$ and $c_{j} \geq 0$ for all $2 \leq j \leq N$. Let $\mathbf{x}^{*}=\left[a_{1}, \ldots, a_{N}\right]^{T}$ where $a_{j}=c_{j} / c_{1}$. Then we have

$$
B \mathbf{x}^{*}=\lambda \mathbf{x}^{*}, \quad a_{j} \geq 0, \quad \text { and } \quad a_{1}=1 .
$$

We now define a mass distribution on $\mathcal{P}^{\partial}$. For each branch $\mathcal{I}_{\mathbf{v}_{k}}$ where $\mathbf{v}_{k} \in \mathcal{V}_{k}^{\partial}$ such that $\left[\mathbf{v}_{k}\right]=\mathcal{T}_{i}^{\partial}$ we let

$$
\mu\left(\mathcal{I}_{\mathbf{v}_{k}}\right):=\lambda^{-k} a_{i} .
$$

(Note that $\mu\left(\mathcal{I}_{\mathbf{v}_{\text {root }}}\right)=\lambda^{0} a_{1}=1$.) We leave it as an exercise to show that $\mu$ is indeed a mass distribution on $\mathcal{P}^{\partial}$.

Next, we transport $\mu$ to a mass distribution on $\partial F$. Recall from Theorem 1.1(c) that

$$
\partial F=\bigcap_{k=0}^{\infty} \bigcup_{\mathbf{v} \in \mathcal{V}_{k}^{\partial}} \phi_{\mathbf{v}}(F) .
$$


Notice that $\phi_{\mathbf{v}^{\prime}}(F) \subseteq \phi_{\mathbf{v}}(F)$ if $\mathbf{v}^{\prime} \in \mathcal{V}_{k+1}^{\partial}$ is an offspring, in $\mathcal{G}_{R}^{\partial}$, of $\mathbf{v} \in \mathcal{V}_{k}^{\partial}$. The expression in (4-2) implies that each path $\left(\mathbf{v}_{0}=\mathbf{v}_{\text {root }}, \mathbf{v}_{1}, \mathbf{v}_{2}, \ldots\right) \in \mathcal{P}^{\partial}$ corresponds to a point $x \in \partial F$, which is the unique point $\bigcap_{k \geq 0} \phi_{\mathbf{v}_{k}}(F)$. We call $\left(\mathbf{v}_{0}, \mathbf{v}_{1}, \ldots\right) \in \mathcal{P}^{\partial}$ an address of $x$. For a subset $U \subseteq \mathbb{R}^{d}$, let $\mathcal{C}(U)$ be the set of all addresses of points in $\partial F \cap U$, and define

$$
\mu^{*}(U):=\mu(\mathcal{C}(U))
$$

Then $\mu^{*}$ is a mass distribution supported on $\partial F$.

Finally, we apply the mass distribution principle by using $\mu^{*}$. Let $0<\delta<\rho$. For any set $U \subseteq \mathbb{R}^{d}$ with $\operatorname{diam}(U) \leq \delta$, assume that $\rho^{k+1} \leq \operatorname{diam}(U)<\rho^{k}$. Lemma 4.1 implies that $U$ can intersect no more than $M$ of the $\phi_{\mathbf{v}}(F)$ with $\mathbf{v} \in \mathcal{V}_{k}^{\partial}$, where $M$ is a fixed constant independent of $k$. Let $\mathbf{v}_{1}, \ldots, \mathbf{v}_{\ell}, \ell \leq M$, be the vertices in $\mathcal{V}_{k}^{\partial}$ such that $U \cap \phi_{\mathbf{v}_{j}}(F) \neq \emptyset$. Then, by $(4-2)$ and the definition of $\mu^{*}$, we have

$$
\begin{aligned}
\mu^{*}(U)=\mu^{*}(U \cap \partial F) & \leq \sum_{j=1}^{\ell} \mu^{*}\left(U \cap \phi_{\mathbf{v}_{j}}(F)\right) \\
& \leq \sum_{j=1}^{\ell} \mu\left(\mathcal{I}_{\mathbf{v}_{j}}\right) \leq M \lambda^{-k} \max _{1 \leq i \leq N}\left\{a_{i}\right\} .
\end{aligned}
$$

Now $\lambda^{-1}=\rho^{s}$ implies that

$$
\lambda^{-k}=\rho^{k s}=\rho^{-s} \rho^{(k+1) s} \leq \rho^{-s}(\operatorname{diam}(U))^{s} .
$$

It follows that

$$
\mu^{*}(U) \leq C(\operatorname{diam}(U))^{s},
$$

where $C=M \rho^{-s} \max _{1 \leq i \leq N}\left\{a_{i}\right\}$. The mass distribution principle implies that

$$
\mathcal{H}^{s}(\partial F) \geq \mu^{*}(\partial F) / C>0,
$$

proving the theorem.

Proof of Theorem 1.2: Theorem 4.4 implies that

$$
\operatorname{dim}_{H}(\partial F) \leq \operatorname{dim}_{B}(\partial F)=s=\frac{\ln \lambda_{B}}{-\ln \rho} .
$$

Theorem 4.5 implies that $\operatorname{dim}_{H}(\partial F) \geq s$. This proves part (a). Part (b) is proved in Theorem 4.5.

The proof of Theorem 1.3 uses a technique in [Ngai and Wang 01, Theorem 1.3].

Proof of Theorem 1.3: Suppose that $\operatorname{dim}_{H}(\partial F)=d$. Then by Theorem 1.2(b), $\mathcal{H}^{d}(\partial F)>0$. Hence, there exists a Lebesgue point $x^{*} \in \partial F$ and

$$
c_{k}:=\frac{\mathcal{H}^{d}\left(\partial F \cap B_{\rho^{k}}\left(x^{*}\right)\right)}{\mathcal{H}^{d}\left(B_{\rho^{k}}\left(x^{*}\right)\right)} \rightarrow 1 \quad \text { as } \quad k \rightarrow \infty,
$$

where $\rho=\min \left\{\rho_{i}: 1 \leq i \leq q\right\}$. By Theorem 1.1(c),

$$
\partial F=\bigcap_{k=0}^{\infty} \bigcup_{\mathbf{v} \in \mathcal{V}_{k}^{\partial}} \phi_{\mathbf{v}}(\partial F) .
$$

Define $\mathcal{U}_{k}:=\left\{\mathbf{v} \in \mathcal{V}_{k}^{\partial}: \phi_{\mathbf{v}}(\partial F) \cap B_{\rho^{k}}\left(x^{*}\right) \neq \emptyset\right\}$. Вy

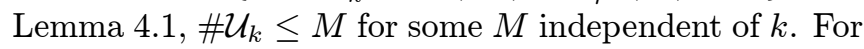
each $k \geq 0$, define an expansive similitude $\tau_{k}: \mathbb{R}^{d} \rightarrow \mathbb{R}^{d}$ by $\tau_{k}(x):=\rho^{-k} x-\rho^{-k} x^{*}$. Then

$$
\mathcal{U}_{k}=\left\{\mathbf{v} \in \mathcal{V}_{k}^{\partial}: \tau_{k} \circ \phi_{\mathbf{v}}(\partial F) \cap B_{1}(0) \neq \emptyset\right\} .
$$

Moreover, by (4-4), $\tau_{k}\left(\partial F \cap B_{\rho^{k}}\left(x^{*}\right)\right) \subseteq \cup_{\mathbf{v} \in \mathcal{U}_{k}}\left(\tau_{k} \circ\right.$ $\left.\phi_{\mathbf{v}}(\partial F) \cap B_{1}(0)\right)$. Define $E_{k}:=\cup_{\mathbf{v} \in \mathcal{U}_{k}} \tau_{k} \circ \phi_{\mathbf{v}}(\partial F)$. Then $\tau_{k}\left(\partial F \cap B_{\rho^{k}}\left(x^{*}\right)\right) \subseteq E_{k} \cap B_{1}(0)$ and by $(4-3)$,

$$
\mathcal{H}^{d}\left(E_{k} \cap B_{1}(0)\right) \geq c_{k} \mathcal{H}^{d}\left(B_{1}(0)\right) .
$$

Choose a subsequence $\left\{E_{k_{j}}\right\}$ of $\left\{E_{k}\right\}$ which converges to a compact set $E$ in the Hausdorff metric. Then, by using the fact that $E=\cap_{n=0}^{\infty} \overline{\cup_{j \geq n} E_{k_{j}}}$, we have

$$
\begin{aligned}
\mathcal{H}^{d}\left(E \cap B_{1}(0)\right) & \geq \lim _{j \rightarrow \infty} \mathcal{H}^{d}\left(E_{k_{j}} \cap B_{1}(0)\right) \\
& \geq \lim _{j \rightarrow \infty} c_{k_{j}} \mathcal{H}^{d}\left(B_{1}(0)\right)=\mathcal{H}^{d}\left(B_{1}(0)\right) .
\end{aligned}
$$

Hence, $E \cap B_{1}(0)=B_{1}(0)$. Now, $E_{k_{j}}$ is a union of no more than $M$ sets $\tau_{k_{j}} \circ \phi_{\mathbf{v}}(\partial F)$, with $\mathbf{v} \in \mathcal{U}_{k_{j}}$ and each $\tau_{k_{j}} \circ \phi_{\mathbf{v}}$ being a similitude with ratio between $\rho$ and 1 . Moreover, (4-5) implies that these $\tau_{k_{j}} \circ \phi_{\mathbf{v}}(\partial F)$ are uniformly bounded. By compactness, we have $E=\cup_{\ell=1}^{L} H_{\ell}$, where $L \leq M$ and each $H_{\ell}$ is the limit of some sequence $\left\{\tau_{k_{j}} \circ \phi_{\mathbf{v}}(\partial F): \mathbf{v} \in \mathcal{U}_{k_{j}}\right\}$. Hence, each $H_{\ell}=\sigma_{\ell}(\partial F)$ for some similitude $\sigma_{\ell}$ on $\mathbb{R}^{d}$. Since $E^{\circ} \neq \emptyset$, we have $H_{\ell}^{\circ} \neq \emptyset$ for some $\ell$. Therefore, $(\partial F)^{\circ} \neq \emptyset$. This contradiction completes the proof.

\section{EXAMPLES ON COMPUTING DIMENSIONS}

In this section, we illustrate the algorithm by some examples. In actual computations, it is often difficult to determine whether a neighborhood type belongs to $\mathcal{N}^{\circ}$ or $\mathcal{N}^{\partial}$. Proposition 5.1 provides some partial results. Let $F$ be the attractor of an IFS $\left\{\phi_{i}\right\}_{i=1}^{q}$ on $\mathbb{R}^{d}$ as defined in (1-1) and let $\Omega \supseteq F$ be a bounded open invariant set. We first define a partial order $\leq$ on $\mathcal{N}$. Let $\mathbf{u}, \mathbf{v} \in \mathcal{V}$ with $\Omega(\mathbf{u})=\left\{\mathbf{u}_{0}, \mathbf{u}_{1}, \ldots, \mathbf{u}_{m}\right\}$ and $\Omega(\mathbf{v})=\left\{\mathbf{v}_{0}, \mathbf{v}_{1}, \ldots, \mathbf{v}_{n}\right\}$, where $\mathbf{u}_{0}=\mathbf{u}, \mathbf{v}_{0}=\mathbf{v}$, and $m \leq n$. We say that $\mathbf{u} \leq \mathbf{v}$ if there exists a similitude $\sigma(x)=\rho^{k} U x+c$, where $k \in \mathbb{Z}$, $U$ is orthogonal and $c \in \mathbb{R}^{d}$, such that

$$
\begin{aligned}
& \sigma \circ \phi_{\mathbf{u}_{0}}=\phi_{\mathbf{v}_{0}} \text { and } \\
& \left\{\sigma \circ \phi_{\mathbf{u}_{i}}: i=0,1, \ldots, m\right\} \subseteq\left\{\phi_{\mathbf{v}_{i}}: i=0,1, \ldots, n\right\} .
\end{aligned}
$$


$\leq$ is a partial order on $\mathcal{V}$. Also, $\leq$ extends naturally to $\mathcal{N}$. We say that a neighborhood type $[\mathbf{v}] \in \mathcal{N}$ is maximal if

$$
[\mathbf{u}] \not \mathbf{v}] \text { for all } \mathbf{u} \notin[\mathbf{v}] \text {. }
$$

Proposition 5.1. Assume the above conditions and let $\mathcal{T}, \mathcal{T}_{1}, \mathcal{T}_{2} \in \mathcal{N}$. The following hold.

(a) $\mathcal{T} \in \mathcal{N}^{\circ}$ if and only if all offspring of $\mathcal{T}$ belong to $\mathcal{N}^{\circ}$. Equivalently, $\mathcal{T} \in \mathcal{N}^{\partial}$ if and only if some offspring of $\mathcal{T}$ belongs to $\mathcal{N}^{\partial}$.

(b) If $\mathcal{T}_{1} \leq \mathcal{T}_{2}$ and $\mathcal{T}_{2} \in \mathcal{N}^{\partial}$, then $\mathcal{T}_{1} \in \mathcal{N}^{\partial}$.

(c) If $\mathcal{T}_{1} \leq \mathcal{T}_{2}$ and $\mathcal{T}_{1} \in \mathcal{N}^{\circ}$, then $\mathcal{T}_{2} \in \mathcal{N}^{\circ}$.

(d) Assume that $\rho_{i}, i=1, \ldots, q$, are exponentially commensurable. If $F^{\circ} \neq \emptyset$ and $\mathcal{T}$ is maximal, then $\mathcal{T} \in \mathcal{N}^{\circ}$.

Proof: (a), (b), and (c) follow from Propositions 2.2 and 2.3. To prove (d), it suffices to show that if $\mathcal{T}=[\mathbf{v}] \in \mathcal{N}^{\partial}$, then $\mathcal{T}$ is not maximal. Let

$\mathbf{v}=\left(\phi_{\mathbf{j}}, k\right)$ and $\Omega(\mathbf{v})=\left\{\mathbf{v}_{i}: i=0,1, \ldots, n\right\}$ with $\mathbf{v}_{0}:=\mathbf{v}$.

Since $F^{\circ} \neq \emptyset$ and the $\rho_{i}$ are exponentially commensurable, there exists some index $\mathbf{i}$ and some $\ell \in \mathbb{N}$ such that

$$
\rho_{\mathbf{i}}=\rho^{\ell} \quad \text { and } \quad \phi_{\mathbf{i}}(\Omega) \subseteq F^{\circ} .
$$

Note that for $j=0,1, \ldots, n, \phi_{\mathbf{i}} \circ \phi_{\mathbf{v}_{j}} \in \mathcal{V}_{\ell+k}$, and $\left(\phi_{\mathbf{i}} \circ\right.$ $\left.\phi_{\mathbf{v}_{j}}, \ell+k\right)$ are neighbors of $\left(\phi_{\mathbf{i}} \circ \phi_{\mathbf{v}_{0}}, \ell+k\right)$. Let $\mathbf{u}=$ $\left(\phi_{\mathbf{i j}}, \ell+k\right)$. Then $\mathcal{T}=[\mathbf{v}] \leq[\mathbf{u}]$. Since $[\mathbf{u}] \in \mathcal{N}^{\circ}, \mathcal{T} \neq[\mathbf{u}]$ and therefore $\mathcal{T}$ is not maximal.

We remark that $\left[\mathbf{v}_{\text {root }}\right] \in \mathcal{N}^{\partial}$ and that the converse of Proposition 5.1(d) is false (see Example 5.3).

Let $\{\phi\}_{i=1}^{q}$ be a finite boundary type IFS of contractive similitudes on $\mathbb{R}^{d}$ and fix an FBT-set $\Omega$. Let $\mathbf{v} \in \mathcal{V}_{k}^{\partial}$ with $[\mathbf{v}]=\mathcal{T}^{\partial}$. Suppose $\mathbf{v}$ generates $n_{j}$ offspring in $\mathcal{V}_{k+1}^{\partial}$ of boundary neighborhood types $\mathcal{T}_{j}^{\partial}, 1 \leq j \leq m$. Then we denote this by

$$
\mathcal{T}^{\partial} \rightarrow n_{1} \mathcal{T}_{1}^{\partial}+n_{2} \mathcal{T}_{2}^{\partial}+\cdots+n_{m} \mathcal{T}_{m}^{\partial} .
$$

By Proposition 3.4, this relation is independent of the choice of the representative $\mathbf{v}$.

The following simple example illustrates the fact that a finite boundary type IFS need not be of finite type.

Example 5.2. Consider the IFS

$$
\phi_{1}(x)=\rho x, \quad \phi_{2}(x)=\rho x+(1-\rho),
$$

where $1 / 2<\rho<(\sqrt{5}-1) / 2 \approx 0.618 \ldots$. Then $\left\{\phi_{1}, \phi_{2}\right\}$ is of finite boundary type.
Proof: Note that $\left\{\phi_{1}, \phi_{2}\right\}$ is not necessarily of finite type. In fact, it is not of finite type if $\rho$ is transcendental. To see this, we first observe that if $\rho$ is transcendental, then the iterates $\phi_{\mathbf{v}}(0), \mathbf{v} \in \mathcal{V}_{k}$, do not overlap. Consequently, for each $k$, there are $2^{k}$ distinct iterates. Now, by the pigeonhole principle, some subinterval of $\{0,1\}$ of length $\rho^{k}$ must contain at least $(2 \rho)^{k}$ distinct iterates. Equivalently, some neighborhood $\Omega(\mathbf{v})$ with $\mathbf{v} \in \mathcal{V}_{k}$ must contain at least $(2 \rho)^{k}$ distinct vertices in $\mathcal{V}_{k}$. Therefore, $\left\{\phi_{1}, \phi_{2}\right\}$ cannot be of finite type. In fact, it does not have the weak separation property (see [Lau and Ngai 99]).

Obviously, the attractor $F=[0,1]$. Also, for any $\epsilon>0, \Omega=(-\epsilon, 1+\epsilon)$ is a bounded open invariant set containing $F$. We will choose $\epsilon>0$ to be sufficiently small, say,

$$
0<\epsilon<\left(1-\rho-\rho^{2}\right) / 2 .
$$

Let $\mathcal{T}_{1}^{\partial}:=\left[\mathbf{v}_{\text {root }}\right] . \quad \mathbf{v}_{\text {root }}$ generates two offspring in $\mathcal{G}_{R}^{\partial}$, namely,

$$
\mathbf{v}_{1}=\left(\phi_{1}, 1\right) \quad \text { and } \quad \mathbf{v}_{2}=\left(\phi_{2}, 1\right) .
$$

Denote $\left[\mathbf{v}_{1}\right]$ and $\left[\mathbf{v}_{2}\right]$ by $\mathcal{T}_{2}^{\partial}$ and $\mathcal{T}_{3}^{\partial}$, respectively. Then

$$
\mathcal{T}_{1}^{\partial} \rightarrow \mathcal{T}_{2}^{\partial}+\mathcal{T}_{3}^{\partial} .
$$

Iterating one more time and using assumption (5-1), we easily get

$$
\mathcal{T}_{2}^{\partial} \rightarrow \mathcal{T}_{2}^{\partial} \quad \text { and } \quad \mathcal{T}_{3}^{\partial} \rightarrow \mathcal{T}_{3}^{\partial} .
$$

Since no new boundary neighborhood types can be generated, we conclude that $\left\{\phi_{1}, \phi_{2}\right\}$ is of finite boundary type. Moreover, the boundary offspring matrix is

$$
B=\left[\begin{array}{lll}
0 & 1 & 1 \\
0 & 1 & 0 \\
0 & 0 & 1
\end{array}\right]
$$

with $\lambda_{B}=1$. Hence, $\operatorname{dim}_{H}(\partial F)=\operatorname{dim}_{B}(\partial F)=$ $\ln 1 /(-\ln \rho)=0$, as expected.

The common contraction ratio of the similitudes in the following example is the reciprocal of a Pisot number.

Example 5.3. Let $F$ be the attractor of the IFS $\left\{\phi_{i}\right\}_{i=1}^{4}$ defined by

$$
\begin{array}{ll}
\phi_{1}(x)=r^{2} x, & \phi_{2}(x)=r^{2} x+r^{2}, \\
\phi_{3}(x)=r^{2} x+r, & \phi_{4}(x)=r^{2} x+\frac{1}{r^{2}},
\end{array}
$$

where $r=(\sqrt{5}-1) / 2$ is the reciprocal of the golden ratio (see Figure 1). Then

$$
\operatorname{dim}_{H}(\partial F)=\operatorname{dim}_{B}(\partial F)=\frac{\log \lambda_{B}}{-\log \left(r^{2}\right)}=0.6007712354 \ldots,
$$




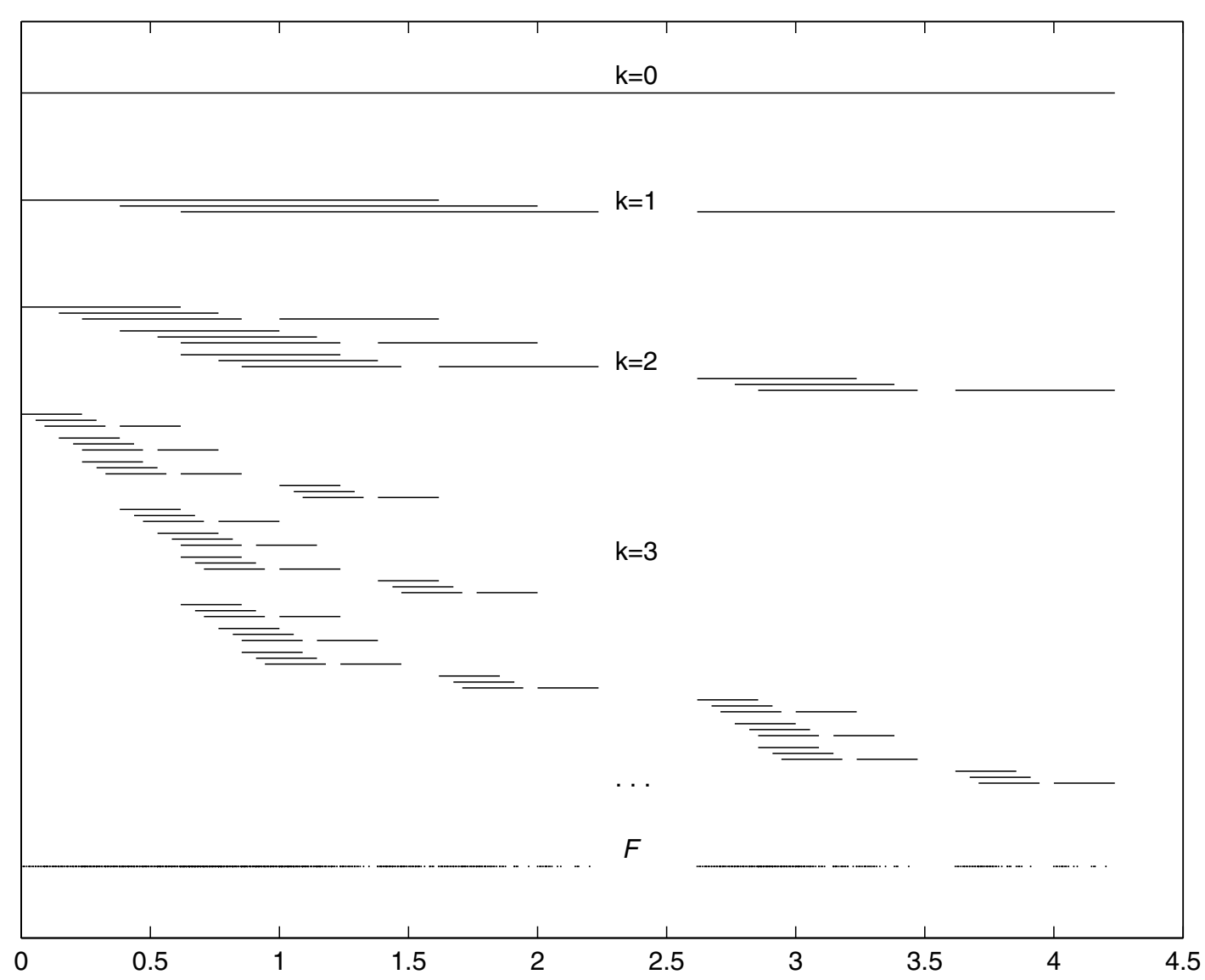

FIGURE 1. The first three iterations of the interval $\left[0, r^{-3}\right]$ under the IFS in Example 5.3 , where $r=(\sqrt{5}-1) / 2$ is the reciprocal of the golden ratio. Vertices are represented by intervals and are slightly separated vertically. The attractor $F$ is shown at the bottom of the figure.

where $\lambda_{B}$ is the largest real zero of the polynomial $p(x)=$ $x^{7}-x^{6}-2 x^{5}+x^{4}+x-1$.

Proof: By Example 3.9, $\left\{\phi_{i}\right\}_{i=1}^{4}$ is of finite boundary type. It can be checked directly that the attractor of the subsystem $\left\{\phi_{1}, \phi_{2}, \phi_{3}\right\}$ is the interval $[0,1]$ and therefore, $(0,1) \subseteq F$; moreover, $F \subseteq\left[0, r^{-3}\right]$. We let $\Omega=\left(-\epsilon, r^{-3}+\right.$ $\epsilon)$ be an FBT-set with $\epsilon=10^{-5}$. We use Mathematica to find all the neighborhood types in $\mathcal{N}$. There are 62 of them. Using Propositions 2.2 and 2.3, we can partition $\mathcal{N}$ into $\mathcal{N}^{\circ} \cup \mathcal{N}^{\partial}$.

First, we use Proposition 2.2 to find as many interior neighborhood types as possible. Note that if $E \subseteq F^{\circ}$, then $\cup_{i=1}^{4} \phi_{i}(E) \subseteq F^{\circ}$. Using this and the fact that $(0,1) \subseteq F^{\circ}$, we can enlarge the known subset of $F^{\circ}$. Unfortunately, the largest subinterval of $F^{\circ}$ that can be found by using this method alone is $(0, a)$, where $a$ can be taken to be 1.236. In fact, $a<1+r^{3}=1.2360679775 \ldots$.
To enlarge this known subinterval of $F^{\circ}$, we will prove the following claim

Claim 5.4. (a) $1+r^{3} \in F^{\circ}$. (b) $1+r^{3}+r^{5}$ (= $1.3262379212 \ldots) \in F^{\circ}$.

Proof: We will prove (a); the proof for (b) is similar. It follows from iterating the $\phi_{i}$ s that $1+r^{3}=\phi_{\mathbf{v}_{0}}(0)$ for some $\mathbf{v}_{0} \in \mathcal{V}_{3}$. Hence, there exists some $\epsilon_{0}>0$ such that $\left(1+r^{3}, \epsilon_{0}\right) \in F^{\circ}$. To show that $1+r^{3} \in F^{\circ}$, we will show that there exists an increasing sequence $\left\{R_{k}\right\}$ such that $\left(0, R_{k}\right) \subseteq F^{\circ}$ and $\lim _{k \rightarrow \infty} R_{k}=1+r^{3}$.

First, a direct iteration shows that $1+r^{3}=\phi_{\mathbf{v}_{1}}(0)$ for some $\mathbf{v}_{1} \in \mathcal{V}_{5}$. Let $\mathcal{T}=\left[\mathbf{v}_{1}\right]$. Upon one more iteration, we notice that $\mathbf{v}_{1}$ generates an offspring $\mathbf{v}_{2}$ which is also of neighborhood type $\mathcal{T}$, and moreover, $\phi_{\mathbf{v}_{2}}(0)=1+r^{3}$ (see Figure 2). $\mathbf{v}_{2}$ has four left neighbors $\mathbf{u}_{1}, \mathbf{u}_{2}, \mathbf{u}_{3}, \mathbf{u}_{4} \in$ $\mathcal{V}_{6}$, with 


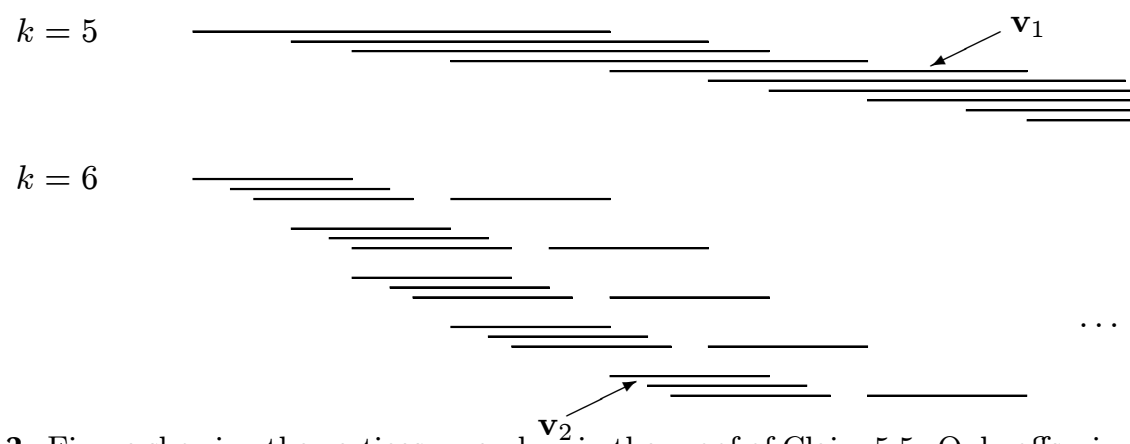

FIGURE 2. Figure showing the vertices $\mathbf{v}_{1}$ and $\mathbf{v}_{2}$ in the proof of Claim 5.5. Only offspring generated by the left neighbors of $\mathbf{v}_{1}$ are shown.

$$
\begin{array}{rrr}
\mathbf{u}_{1}(0)=1+r^{3}-r^{9}, & \mathbf{u}_{2}(0)=1+r^{3}-r^{9}+r^{12}, \\
\mathbf{u}_{3}(0)=1+r^{3}-r^{10}, & \mathbf{u}_{4}(0)=1+r^{3}-r^{11} .
\end{array}
$$

Let $R_{5}=1.236$. Then we know from above that $\left(0, R_{5}\right) \subseteq$ $F^{\circ}$. Iterations from $k=5$ to $k=6$ show that for

$$
R_{6}:=1+r^{3}-r^{11}+r^{12} R_{5}=1+r^{3}-r^{11}\left(1-r R_{5}\right),
$$

we have $\left(0, R_{6}\right) \subseteq F^{\circ}$. In general, for $k \geq 5$, let

$$
R_{k+1}:=1+r^{3}-r^{2 k-1}\left(1-r R_{k}\right) .
$$

Then $\left(0, R_{k+1}\right) \subseteq F^{\circ}$. It can be proved by induction that $1<R_{k}<1+r^{3}$ for all $k \geq 5,\left\{R_{k}\right\}_{k=5}^{\infty}$ is increasing, and $\lim _{k \rightarrow \infty} R_{k}=1+r^{3}$. This proves Claim 5.4.

We remark that $\left[\mathbf{v}_{0}\right],\left[\mathbf{v}_{1}\right],\left[\mathbf{v}_{2}\right]$ above are not maximal. Claim 5.4 allows us to compute a larger subinterval $(0, b) \subseteq F^{\circ}$; in fact, $b$ can be taken to be at least 1.339 . The following iterates of $(0, b)$ also belong to $F^{\circ}$ :

$$
\begin{array}{ll}
1+r^{2}+r^{4}(0, b), & 1+r+r^{4}(0, b), \\
1+r^{3}+r^{6}(0, b), & 1+2 r^{2}+r^{6}(0, b),
\end{array}
$$

Combining this with Proposition 2.2, 31 of the 62 neighborhood types are found to belong to $\mathcal{N}^{\circ}$.

We now try to find as many boundary neighborhood types as possible. First, notice that if a vertex $\mathbf{v}$ does not have either a left neighbor or a right neighbor, or has a left neighbor $\mathbf{u}$ such that $\phi_{\mathbf{u}}(F)$ intersects $\phi_{\mathbf{v}}(F)$ only at the left endpoint of $\phi_{\mathbf{v}}(F)$, then $[\mathbf{v}] \in \mathcal{N}^{\partial}$. There are nine such neighborhood types.

Next, using the fact that any vertex that eventually generates a boundary vertex is a boundary vertex (Proposition 5.1), 19 additional neighborhood types are found to belong to $\mathcal{N}^{\partial}$. This leaves us with three undetermined neighborhood types; each of them can be identified with a vertex $\mathbf{v}$ such that $\phi_{\mathbf{v}}(0)=1+r$. The following claim shows that they all belong to $\mathcal{N}^{\partial}$.
Claim 5.5. $1+r \in \partial F$.

Proof of Claim 5.5: Starting from the $k$-th iteration with $k \geq 4$, the vertex $\mathbf{v}$ on $\mathcal{V}_{k}$ such that $\phi_{\mathbf{v}}(0)=1+r$ is of two possible neighborhood types, say $\mathcal{T}_{1}$ and $\mathcal{T}_{2}$. More precisely, $[\mathbf{v}]=\mathcal{T}_{1}$ if $k$ is even and $[\mathbf{v}]=\mathcal{T}_{2}$ if $k$ is odd. It can be seen directly that if $k$ is odd, then $\mathbf{v}$ always has a left neighbor which is known to belong to $\mathcal{V}^{\partial}$. Hence, there exists a sequence of points in $\partial F$ converging to $1+r$, proving Claim 5.5.

Claim 5.5 completes the characterization of the 62 neighborhood types into 31 interior and 31 boundary neighborhood types. The maximal eigenvalue of the boundary offspring matrix is given by the largest real zero of the polynomial $p(x)$. The Hausdorff dimension of $\partial F$ can now be computed by using Theorem 1.2.

There is an alternative way to determine the eigenvalue $\lambda_{B}$ if the IFSs is of finite type. We first construct the offspring matrix $A$ for all the neighborhood types in $\mathcal{N}$ as described in [Ngai and Wang 01]. The matrix $A$ can be rearranged in a lower block triangular form with each square block on the diagonal being irreducible. By Proposition 5.1, all neighborhood types corresponding to each irreducible block belong to either $\mathcal{N}^{\partial}$ or $\mathcal{N}^{\circ}$, but not both. $\lambda_{B}$ is the maximum of the eigenvalues of the irreducible blocks corresponding to neighborhood types in $\mathcal{N}^{\partial}$. This method is useful when it is difficult to determine a subset of $F^{\circ}$, as in the following example. We have also applied this method to the IFSs in Example 5.3 and [He et al. 03, Example 5.5]. We remark that, unlike the situation in [Veerman 98], $\lambda_{B}$ is not necessarily the second maximal eigenvalue of $A$.

Example 5.6. Let $F \subseteq \mathbb{R}^{2}$ (see Figure $3(\mathrm{a})$ ) be the attractor of the IFS $\phi_{i}(\mathbf{x})=\rho R(\pi) \mathbf{x}+b_{i}, i=1,2,3,4$, 


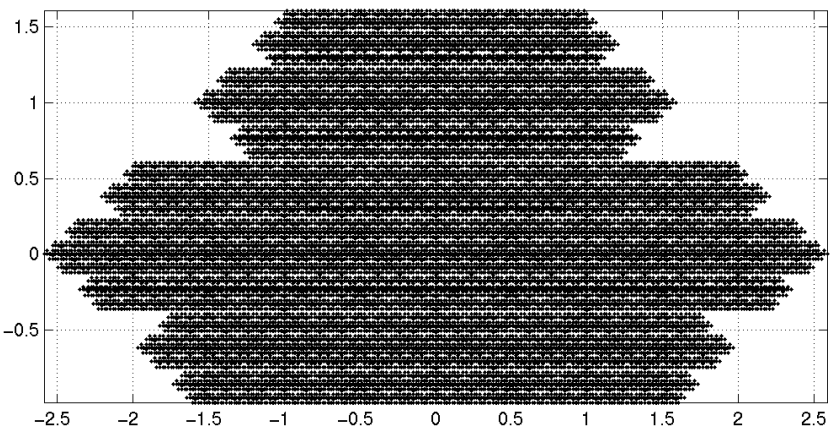

(a)

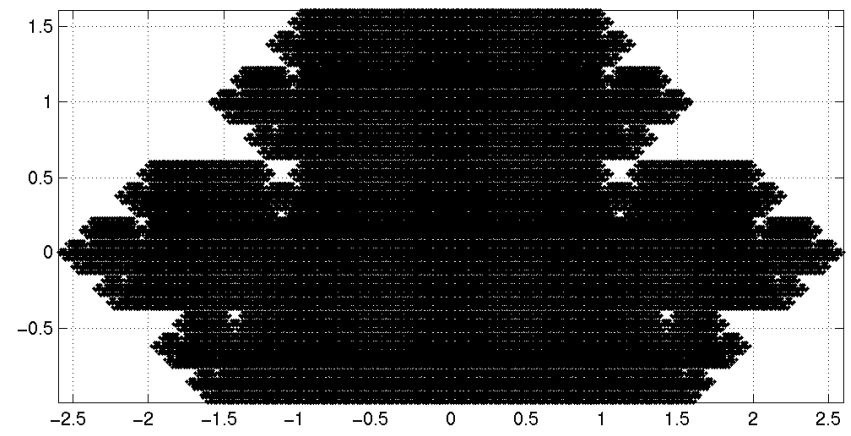

(b)

FIGURE 3. (a) The attractor $F$ in Example 5.6. (b) The attractor $F$ in Example 5.7.

where $\rho=(\sqrt{5}-1) / 2, R(\pi)$ is a counterclockwise rotation by $\pi, b_{1}=(0,0), b_{2}=(-1,0), b_{3}=(1,0)$, and $b_{4}=(0,1)$. Then $F^{\circ} \neq \emptyset, \lambda_{B}=\rho^{-1}$ and $\operatorname{dim}_{B}(\partial F)=$ $\operatorname{dim}_{H}(\partial F)=1$.

Proof: Again by Example 3.9, $\left\{\phi_{i}\right\}_{i=1}^{4}$ is of finite boundary type. Since $F$ is enclosed in the rectangle $[-2-\rho, 2+$ $\rho] \times[-1,1+\rho]$, we let $\Omega=(-2-\rho-\epsilon, 2+\rho+\epsilon) \times(-1-$ $\epsilon, 1+\rho+\epsilon)$, with some small $\epsilon>0$. The IFS is of finite type and the maximal eigenvalue of the offspring matrix $A$ is $\rho^{-2}$. It follows that $\operatorname{dim}_{H}(F)=2$ and therefore by Theorem 1.3, $F^{\circ} \neq \emptyset$. To compute the dimensions of $\partial F$, we first use MATLAB to find all irreducible components of $A$. The one with maximal eigenvalue $\rho^{-2}$ must consist entirely of neighborhood types in $\mathcal{N}^{\circ}$. The maximum of the maximal eigenvalues of the remaining irreducible components is $\rho^{-1}$ (the larger zero of $x^{2}-x-1$ ). It is easy to check that one of these irreducible components contains a neighborhood type in $\mathcal{N}^{\partial}$. Hence, $\lambda_{B}=\rho^{-1}$, and the result follows.

Note that in Example 5.6, $F=\cup_{i, j=1}^{4} \phi_{i} \circ \phi_{j}(F)$. By removing two of the maps $\phi_{i} \circ \phi_{j}$, we obtain, in the following example, an attractor whose boundary has Hausdorff dimension exceeding one. The computation is similar.

Example 5.7. Let $\left\{\phi_{i}\right\}_{i=1}^{4}$ be as in Example 5.6 and let $F$ be the attractor of the IFS $\left\{\phi_{i} \circ \phi_{j}\right\}_{i, j=1}^{4} \backslash\left\{\phi_{2} \circ \phi_{1}, \phi_{3} \circ \phi_{1}\right\}$ (see Figure 3(b)). Then $F^{\circ} \neq \emptyset, \lambda_{B}$ is the large real zero of $x^{5}-3 x^{4}-x^{3}+x^{2}+5 x-1$, and $\operatorname{dim}_{B}(\partial F)=$ $\operatorname{dim}_{H}(\partial F)=1.1608863490 \ldots$.

\section{ACKNOWLEDGMENTS}

Part of this work was carried out while the second author was visiting the Department of Mathematics of the Chinese
University of Hong Kong. He thanks the Department for its hospitality and support. The authors thank Professors John R. Gilbert and Cleve Moler for their help with MATLAB.

The first author is supported in part by an HKRGC grant. The second author is supported in part by an HKRGC grant, a Faculty Research Grant from Georgia Southern University, and a Foundation Grant from the Department of Mathematics and Computer Science of Georgia Southern University.

\section{REFERENCES}

[Dekking and van der Wal 02] F. M. Dekking and P. van der Wal. "The Boundary of the Attractor of a Recurrent Iterated Function System." Fractals 10:1 (2002), 77-89.

[Duvall et al. 00] P. Duvall, J. Keesling and A. Vince. "The Hausdorff Dimension of the Boundary of a Self-Similar Tile." J. London Math. Soc. (2) 61 (2000), 748-760.

[Falconer 90] K. J. Falconer. Fractal Geometry: Mathematical Foundations and Applications. Chichester: John Wiley \& Sons, Ltd., 1990.

[He et al. 03] X.-G. He, K.-S. Lau, and H. Rao. "Self-Affine Sets and Graph-Directed Systems." Constructive Approx. 19 (2003), 373-397.

[Hutchinson 81] J. E. Hutchinson. "Fractals and SelfSimilarity." Indiana Univ. Math. J. 30 (1981), 713-747.

[Keesling 99] J. Keesling. The Boundaries of Self-Similar Tiles in $\mathbb{R}^{n}$." Topology Appl. 94 (1999), 195-205.

[Kenyon et al. 99] R. Kenyon, J. Li, R. S. Strichartz, and Y. Wang. "Geometry of Self-Affine Tiles II." Indiana Univ. Math. J. 48 (1999), 25-42.

[Lagarias and Wang 96] J. C. Lagarias and Y. Wang. "SelfAffine Tiles in $\mathbb{R}^{n}$." Adv. Math. 121 (1996), 21-49.

[Lau and Ngai 99] K.-S. Lau and S.-M. Ngai. "Multifractal Measures and a Weak Separation Condition." Adv. Math. 141 (1999), 45-96. 
[Lau and Xu 00] K.-S. Lau and Y. Xu. "On the Boundary of Attractors with Non-Void Interior." Proc. Amer. Math. Soc. 128 (2000), 1761-1768.

[Ngai and Wang 01] S.-M. Ngai and Y. Wang. "Hausdorff Dimension of Self-Similar Sets with Overlaps." J. London Math. Soc. (2) 63 (2001), 655-672.
[Strichartz and Wang 99] R. S. Strichartz and Y. Wang. "Geometry of Self-Affine Tiles I." Indiana Univ. Math. J. 48 (1999), 1-23.

[Veerman 98] J. J. P. Veerman. "Hausdorff Dimension of Boundaries of Self-Affine Tiles in $\mathbf{R}^{N}$." Bol. Soc. Mat. Mexicana (3) 4 (1998), 159-182.

Ka-Sing Lau, Department of Mathematics, The Chinese University of Hong Kong, Shatin, NT, Hong Kong (kslau@math.cuhk.edu.hk)

Sze-Man Ngai, Department of Mathematical Sciences, Georgia Southern University, Statesboro, GA 30460-8093, United States (ngai@gsu.cs.gasou.edu)

Received January 3, 2003; accepted April 29, 2003. 